The Astrophysical Journal, 662:472-486, 2007 June 10

(C) 2007. The American Astronomical Society. All rights reserved. Printed in U.S.A.

\title{
ARE THE MODELS FOR TYPE Ia SUPERNOVA PROGENITORS CONSISTENT WITH THE PROPERTIES OF SUPERNOVA REMNANTS?
}

\author{
Carles Badenes, ${ }^{1,2}$ John P. Hughes, ${ }^{1,3}$ Eduardo Bravo, ${ }^{4}$ and Norbert Langer ${ }^{5}$ \\ Received 2007 January 19; accepted 2007 March 12
}

\begin{abstract}
We explore the relationship between the models for progenitor systems of Type Ia supernovae and the properties of the supernova remnants that evolve after the explosion. Most models for Type Ia progenitors in the single-degenerate scenario predict substantial outflows during the presupernova evolution. Expanding on previous work, we estimate the imprint of these outflows on the structure of the circumstellar medium at the time of the supernova explosion, and the effect that this modified circumstellar medium has on the evolution of the ensuing supernova remnant. We compare our simulations with the observational properties of known Type Ia supernova remnants in the Galaxy (Kepler, Tycho, SN 1006), the Large Magellanic Cloud (0509-67.5, 0519-69.0, N103B), and M31 (SN 1885). We find that optically thick outflows from the white dwarf surface (sometimes known as "accretion winds") with velocities above $200 \mathrm{~km} \mathrm{~s}^{-1}$ excavate large low-density cavities around the progenitors. Such large cavities are incompatible with the dynamics of the forward shock and the X-ray emission from the shocked ejecta in all the Type Ia remnants that we have examined.
\end{abstract}

Subject headings: binaries: close — hydrodynamics — supernova remnants — supernovae: general — X-rays: ISM

\section{INTRODUCTION}

Despite decades of continuing effort, the progenitor systems of Type Ia supernovae ( $\mathrm{SNe}$ ) have not been confidently identified yet (see Hillebrandt \& Niemeyer 2000 and references therein). This has become one of the most pressing problems in stellar evolution, with profound implications for cosmology and the chemical evolution of galaxies (Kobayashi et al. 1998). It has been known for some time that Type Ia SNe are the result of the thermonuclear explosion of a $\mathrm{C}+\mathrm{O}$ white dwarf (WD) that is destabilized by accretion of matter from a companion in a close binary system (Hoyle \& Fowler 1960), but the details of this process are still the subject of heated debate. In particular, the nature of the companion and the physical processes involved in the evolution of the binary prior to explosion remain obscure.

Most of the discussions on Type Ia progenitor systems are held in the framework of two basic scenarios. In the so-called singledegenerate (SD) scenario, the WD companion is a normal or slightly evolved star. In the double-degenerate (DD) scenario, it is another WD. In the SD scenario, the WD might explode either close to the Chandrasekhar limit (SD-Ch) or some time before reaching it (sub-Chandrasekhar explosions, SD-subCh). Several observational and theoretical arguments point to SD-Ch explosions as the most promising route to Type Ia SNe, but there are still many uncertainties associated with this paradigm (for discussions, see Branch et al. 1995; Tout 2005). The main problem is the necessity of bringing the mass of the $\mathrm{C}+\mathrm{O}$ WD close enough to

\footnotetext{
${ }^{1}$ Department of Physics and Astronomy, Rutgers University, 136 Frelinghuysen Road, Piscataway, NJ 08854-8019; badenes@physics.rutgers.edu, jph@physics .rutgers.edu.

2 Chandra Fellow.

3 Department of Astrophysical Sciences, Princeton University, Princeton, NJ 08544 .

${ }^{4}$ Departament de Física i Enginyeria Nuclear, Universitat Politècnica de Catalunya, Diagonal 647, Barcelona 08028, Spain; and Institut d'Estudis Espacials de Catalunya, Campus UAB, Facultat de Ciències, Torre C5, Bellaterra, Barcelona 08193, Spain; eduardo.bravo@upc.es.

5 Astronomical Institute, University Utrecht, P.O. Box 80000, 3508TA Utrecht, Netherlands; n.langer@astro.uu.nl.
}

the Chandrasekhar limit to trigger the explosion. At the onset of the final mass transfer episode in SD systems, the WD mass is thought to lie between $0.7 M_{\odot}$ (Langer et al. 2000; Han \& Podsiadlowski 2004) and $1.2 M_{\odot}$ (Nomoto 1982). This means that, in order to achieve the $\sim 1.38 M_{\odot}$ necessary for a thermonuclear runaway, the WD needs to accrete between 0.2 and $0.7 M_{\odot}$ of material from its companion and then burn it somehow to $\mathrm{C}$ and $\mathrm{O}$, avoiding the highly unstable degenerate conditions at the WD surface. Our present understanding of Type Ia SNe in the SD-Ch scenario is based on theoretical models that attempt to explain the details of these accretion and burning processes.

One key aspect of such models is the presence or absence of outflows from the binary system during its pre-SN evolution. If these outflows are indeed present, they should leave some kind of imprint on the circumstellar medium (CSM) of the progenitor, but the evidence for this modified CSM in Type Ia SN observations remains inconclusive. Prompt emission at radio or X-ray wavelengths from the interaction of SN ejecta with surrounding material, which has been found in several core-collapse SNe, appears to be absent in Type Ia events. In the radio, Panagia et al. (2006) report no VLA detections in 27 nearby objects. In X-rays, no Type Ia SNe have been detected with ROSAT (upper limits on one object; Schlegel \& Petre 1993) or Chandra (upper limits on three objects; J. P. Hughes et al. 2007, in preparation). Immler et al. (2006) report Swift observations of eight Type Ia SNe, with only one marginal $(\sim 3 \sigma)$ detection, SN $2005 \mathrm{ke}$, a peculiar object that was not seen by Chandra. It is important to keep in mind that these studies only probe the CSM at distances between $10^{15} \mathrm{~cm}$ (for observations weeks or months after the explosion) and $10^{17} \mathrm{~cm}$ (for the latest radio observations in Panagia et al. [2006], several years after the explosion). Modification of the CSM on larger scales will become apparent in the aftermath of the explosion, during the supernova remnant (SNR) phase. In Badenes \& Bravo (2001, hereafter BB01), we conducted a preliminary search for the imprint of presupernova outflows on the dynamics of young Type Ia SNRs. In the present work, we expand and revise the calculations presented in BB01, and we perform a more detailed and robust comparison to the observations of SNRs. Our aim is to 
use these comparisons as a means to constrain the fundamental properties of the presupernova outflows and gain insights on the nature of the progenitor systems.

This paper is organized as follows. First, we examine the outflow mechanisms from Type Ia progenitors in the SD-Ch channel, and we build simple models to mimic them $(\S 2)$. From these outflow models, we simulate the structure of the CSM around the progenitor system at the time of the SN explosion $(\S 3)$ and the subsequent evolution of the SNR ( $₫ 4)$. In $\S 5$ we compare our SNR models with the known properties of the forward-shock dynamics and X-ray emission in a sample of known Type Ia SNRs. Finally, we discuss our results in $\S 6$ and we summarize them in $\S 7$.

\section{TYPE Ia PROGENITORS IN THE SINGLE DEGENERATE CHANNEL: PRESUPERNOVA OUTFLOWS}

\subsection{Pre-SN Evolution Scenarios}

There are two channels by which a SD binary system can lead to a Type Ia SN. The systems in the first channel consist of a WD and a main-sequence (MS) or slightly evolved companion star (WD+MS channel; Li \& van den Heuvel 1997; Hachisu et al. 1999b). Mass transfer occurs via Roche lobe overflow, which requires that the two components be very close, with an initial period of the order of days. A different class of Type Ia progenitor systems is composed of a WD and a red giant (RG) star with a wider separation and a longer initial period, of the order of a hundred days (WD+RG channel; Li \& van den Heuvel 1997; Hachisu et al. 1999a). The mechanism for mass transfer in these more detached systems is not well understood, but Roche lobe overflow might still play a significant role.

The details of the evolution of Type Ia progenitor systems in these two channels are very complex. This is mainly due to the fact that the process of mass transfer affects both the orbital parameters of the system and the evolution of the components, which in turn modify the conditions for the mass transfer. In the most widely accepted picture for SD Type Ia progenitors, the H- or He-rich material from the donor star forms an accretion disk and then accumulates in a shell on top of the WD. Given the appropriate conditions, nuclear burning in this shell converts the accreted material to $\mathrm{C}$ and $\mathrm{O}$, until the mass of the WD reaches $\sim 1.38 M_{\odot}$. At this point, a runaway thermonuclear flame ignites in the central regions of the WD, and a Type Ia SN explosion ensues. It is important to stress that a SN explosion is only one of the many possible outcomes of the evolution of close-binary systems that contain a WD. The key parameter that determines whether a specific binary system leads to a Type Ia SN or not is the accretion rate of the WD, $\dot{M}$.

For accretion rates below a critical limit $\dot{M}_{\text {stable, nuclear burn- }}$ ing on the surface of the degenerate WD is unstable (Nomoto 1982). ${ }^{6}$ The value of $\dot{M}_{\text {stable }}$ depends on the mass of the WD (Nomoto \& Kondo 1991), on the metallicity of the accreted material (Piersanti et al. 2000), and on the rotation of the WD (Uenishi et al. 2003; Yoon et al. 2004a, 2004b), but it is generally assumed to be of the order of $10^{-7} M_{\odot} \mathrm{yr}^{-1}$. If $\dot{M}$ is only slightly lower than $\dot{M}_{\text {stable }}$, nuclear burning proceeds cyclically in weak flashes, and most of the accumulated material might be retained by the WD (Kato \& Hachisu 2004). Recurrent novae like RS Oph or U Sco are thought to be the observational counterparts of WD binaries accreting in this regime (Hachisu \& Kato 2001).

\footnotetext{
${ }^{6}$ In a recent paper, Starrfield et al. (2004) proposed a stable accretion regime for low values of $\dot{M}$, but see Nomoto et al. (2007).
}

For even lower values of $\dot{M}$, however, the flashes become stronger, effectively turning into nova explosions that erode the WD (Yaron et al. 2005). If the accretion enters this regime, the WD mass effectively decreases with time and the system cannot produce a Type Ia SN (see Fig. 5 in Kahabka \& van den Heuvel 1997 and references therein).

At higher accretion rates, the energy input from the shell nuclear burning can lead to the expansion of the outer layers of the WD to red giant dimensions (Nomoto \& Kondo 1991). This occurs when $\dot{M}$ approaches the nuclear Eddington accretion rate $\dot{M}_{\text {Edd,nuc }}=L_{\text {Edd }} / \epsilon_{\text {nuc }}$, where $L_{\text {Edd }}=4 \pi c G M / \kappa$ is the Eddington luminosity of the WD, and $\epsilon_{\text {nuc }} \simeq 7 \times 10^{18} \mathrm{erg} \mathrm{g}^{-1}$ is the energy gained by fusing $\mathrm{H}$ into He. For a WD of one solar mass, $\dot{M}_{\text {Edd,nuc }} \simeq 6 \times 10^{-7} M_{\odot} \mathrm{yr}^{-1}$. On expansion of the WD, the binary system may enter a common-envelope phase, which might lead to a spiral-in (due to dynamical friction) and the merging of both components (Iben \& Livio 1993; Ivanova \& Taam 2004). For a long time, it was thought that this would prevent many SD systems from becoming Type Ia progenitors, but Hachisu et al. (1996, hereafter HKN96) proposed a mechanism to avoid the common-envelope phase. These authors claimed that for high values of $\dot{M}$, the luminosity from the shell nuclear burning can drive an optically thick "accretion wind" outflow from the WD surface with an outflow mass-loss rate $\dot{M}_{\text {of }}$ such that the effective value of $\dot{M}$ remains below $\dot{M}_{\text {Edd, nuc. This accretion wind results }}$ in the loss of material from the binary system.

\subsection{Accretion Wind Outflows from Type Ia Progenitor Systems}

In recent times, most of the models for SD Type Ia SN progenitors have relied to a greater or lesser extent on the accretion wind mechanism of HKN96 as a means to limit the effective value of $\dot{M}$. This is despite the fact that several fundamental aspects of the accretion wind mechanism are not well understood. In particular, some kind of self-regulation is required so that $\dot{M}$ falls below $\dot{M}_{\text {Edd, nuc }}$ (to avoid the expansion of the WD), but at the same time remains very close to this critical value (in order for the WD to gain enough mass to explode as a Type Ia SN). Furthermore, the accretion wind is supposed to remain active up to mass transfer rates of the order of the Eddington accretion rate $\dot{M}_{\text {Edd, acc }}=L_{\text {Edd }} / \epsilon_{\text {acc }}$, where $\epsilon_{\text {acc }}=G M / R$ is the specific potential energy gained by accreting matter onto a WD with radius $R$. In this situation $\dot{M}_{\text {of }} \simeq \dot{M}_{\text {Edd, acc }}$, which implies, for wind velocities similar to the WD escape velocity (see $\S 2.2 .1$ ), a ratio of wind to photon momentum of $\eta=1 / c[2 R \kappa /(G M)]^{1 / 2} \sim 10^{2}$. It is known that winds driven by photon scattering in lines cannot work in this regime (Lucy \& Abbott 1993). In principle, continuumdriven winds might reach such high efficiencies, as claimed by HKN96 (see also Owocki et al. 2004; Smith \& Owocki 2006), but this has never been proved with detailed simulations. Due to these and other concerns, the role that accretion winds play in the presupernova evolution of Type Ia progenitors remains controversial. Some authors claim that this mechanism cannot work in a realistic scenario, and that $\mathrm{H}$-accreting WDs cannot explode as Type Ia SNe (Cassisi et al. 1998; Piersanti et al. 1999, 2000).

\subsubsection{Theoretical Studies}

Following the work of HKN96, Hachisu et al. (1999b) explored several specific cases within the WD+MS channel, making use of analytical formulae to follow the evolution of the binary system parameters and mass transfer rates. In a companion paper, Hachisu et al. (1999a) applied the same techniques to systems in the $\mathrm{WD}+\mathrm{RG}$ channel. In these papers, substantial outflows from the Type Ia progenitors were found in all cases. The typical timescales for the outflows are several times $10^{5} \mathrm{yr}$, with mass-loss 
rates $\dot{M}_{\text {of }}$ of the order of $10^{-6} M_{\odot} \mathrm{yr}^{-1}$. In some binary systems, the WD explodes as a Type Ia SN while the optically thick accretion wind is still active. In other cases, the outflow stops some time before the explosion, leading to a more or less extended mass-conservative phase.

Other studies have followed the evolution of binary systems with accretion winds, relying on stellar evolution codes instead of the analytical formulae used by Hachisu et al. (1999a, 1999b). This kind of calculation is necessary in order to estimate the mass transfer rates in the system correctly. In general, stellar evolution codes predict lower values of $\dot{M}_{\text {of }}$ and hence longer evolutionary timescales than those obtained using analytical formulae. The first such study was published by Li \& van den Heuvel (1997), but a more detailed and updated exploration of the parameter space (with $\sim 2300$ binary systems) can be found in Han $\&$ Podsiadlowski (2004). These works have in common that they found substantial outflows from the Type Ia progenitors in all cases. In contrast, Langer et al. (2000) only found outflows in about one-third of the progenitor systems of solar metallicity that they simulated. This is due to more optimistic assumptions concerning the mass accumulation efficiency in accreting WDs, which might be justified by models that include the effect of rotation (Yoon et al. 2004a). It is also important to mention that Langer et al. stopped their simulations for systems that required outflows with $\dot{M}_{\text {of }}>3 \dot{M}_{\text {Edd, nuc }}$.

In the theoretical framework laid down by HKN96, accretion winds are formed in the WD envelope with photospheric velocities of $\sim 1000 \mathrm{~km} \mathrm{~s}^{-1}$ (Hachisu et al. 1999a, 1999b). These high outflow velocities are necessary because the escape velocity from the surface of the WD is also very high (roughly of the same order of magnitude; see Fig. 4 and accompanying discussion in Kato \& Hachisu 1994). The orbital velocities in Type Ia progenitor systems are much lower than this, of the order of $200 \mathrm{~km} \mathrm{~s}^{-1}$ (Langer et al. 2000).

\subsubsection{Observations}

It is useful to compare the theoretical accretion wind models described in the previous section to the observations of binary systems that have been proposed as Type Ia progenitor candidates. The list of candidate objects is very heterogeneous and includes transient supersoft X-ray sources such as RX J0513.96951 (Hachisu \& Kato 2003b), eclipsing binaries with intermittent X-ray emission such as V Sagittae (Hachisu \& Kato 2003a), and recurrent novae such as RS Oph (Hachisu \& Kato 2001) and U Sco (Hachisu et al. 2000).

Among these objects, RX J0513.9-6951 and V Sagittae are of particular interest, because they have nonexplosive outflows that can be modeled using the accretion wind mechanism (Hachisu \& Kato 2003a, 2003b). In the case of RX J0513.9-6951, the outflows have a velocity of $\sim 3800 \mathrm{~km} \mathrm{~s}^{-1}$, with strong hints of a bipolar structure (Pakull et al. 1993; Hutchings et al. 2002). The outflows from V Sagittae are more complex, but a highvelocity component at $\sim 1500 \mathrm{~km} \mathrm{~s}^{-1}$ also appears to be present (Wood \& Lockley 2000). These high outflow velocities support the theoretical values discussed in the previous section. It is interesting to note that both systems are variable sources at optical and X-ray wavelengths, with periods of the order of a few hundred days. Hachisu \& Kato (2003b) attribute the variability of RX J0513.9-6951 to the impact of the strong $\left(\sim 2 \times 10^{-6} M_{\odot} \mathrm{yr}^{-1}\right)$ accretion winds from the WD atmosphere on the surface of the donor star, whose outer envelope is stripped off, temporarily shutting down the accretion process and interrupting the accretion wind itself. The same authors propose a somewhat similar model for V Sagittae, but it is unclear whether this kind of vari- ability should be ubiquitous among binary systems with accretion winds.

\subsection{Modeling the Outflows}

Based on the theoretical studies described in $\S 2.2 .1$, we have produced several models for the outflows from Type Ia SN progenitor systems. The temporal evolution of the outflow mass-loss rate $\dot{M}_{\text {of }}$ is shown in Figure 1, and the fundamental properties of the models are listed in Table 1. Model H1 mimics the mass loss of the example system discussed in $\S 3.5$ of Hachisu et al. (1999b; see their Fig. 7). Model LV1 is built after the example in Figure 1 of Li \& van den Heuvel (1997). Models HP1, HP2, and HP3 are approximations to the outflows from the three representative Type Ia progenitor systems discussed in $\S 3$ of Han \& Podsiadlowski (2004; their Fig. $1 a$ for model HP1, Fig. $1 c$ for model HP2, and Fig. $1 e$ for model HP3). Finally, models L1 and L2 correspond to the binary systems numbers 2 and 31 in Table 2 of Langer et al. (2000) (see their Fig. 7 for model L1 and Fig. 34 in Deutschmann [1998] for model L2).

These outflow models are chosen as representative examples of the typical evolution of Type Ia progenitors of solar metallicity. With them, we expand and improve on the work presented in BB01, aiming at a more complete exploration of the parameter space for Type Ia progenitor systems. Our outflow models are evidently very simplified, but we believe that they capture the essence of the relationship established between the current models for Type Ia progenitor systems and the CSM (a more detailed discussion of some of the issues concerning the outflow models is deferred to $\S 6.1$ ). We note that although we have sampled binary system calculations with different initial conditions and from different authors, the timescales and mass-loss rates of all the outflows are very similar, and should shape the CSM and influence the evolution of the SNRs in a similar way.

\section{THE SHAPING OF THE CIRCUMSTELLAR MEDIUM}

\subsection{Method and Parameters}

We have performed hydrodynamic simulations to calculate the impact that the outflow models presented in $\S 2.3$ would have on the structure of the CSM around the Type Ia progenitors at the time of the SN explosion. For this purpose, we have used VH-1, a numerical hydrodynamics code developed at the University of Virginia by J. Hawley, J. Blondin, and collaborators. VH-1 is based on the piecewise parabolic method of Colella \& Woodward (1984), and the code has been extensively tested and validated in multiple astrophysical scenarios. For the timescales and velocities involved in the outflows from Type Ia SN progenitors, radiative cooling is dynamically important. We have included this process through the standard cooling curves of Sutherland \& Dopita (1993) for temperatures above $10^{4} \mathrm{~K}$, and the values of Dalgarno \& McCray (1972) for $10^{2} \mathrm{~K}<T<10^{4} \mathrm{~K}$ (for a discussion of the cooling curves, see Raga et al. 1997). The simulations are performed in one dimension with spherical symmetry, and they do not include thermal conduction.

Once a particular outflow model is selected, the only parameters that need to be adjusted in our calculations are the outflow velocity $v_{\text {of }}$ and the density and temperature of the interstellar medium (ISM), $\rho_{\text {ISM }}$ and $T_{\text {ISM. }}$. Following the discussion in $\S \S 2.2 .1$ and 2.2.2, we have chosen a reference value of $1000 \mathrm{~km} \mathrm{~s}^{-1}$ for $v_{\text {of }}$. For the ISM parameters $\rho_{\text {ISM }}$ and $T_{\text {ISM }}$ we have chosen reference values typical of the warm atomic phase of the ISM: $\rho_{\text {ISM }}=10^{-24} \mathrm{~g} \mathrm{~cm}^{-3}$ and $T_{\text {ISM }}=10^{4} \mathrm{~K}$ (Ferrière 2001). After a brief theoretical discussion $(\S 3.2)$, we present the CSM structures generated by the outflow models using these fiducial values 

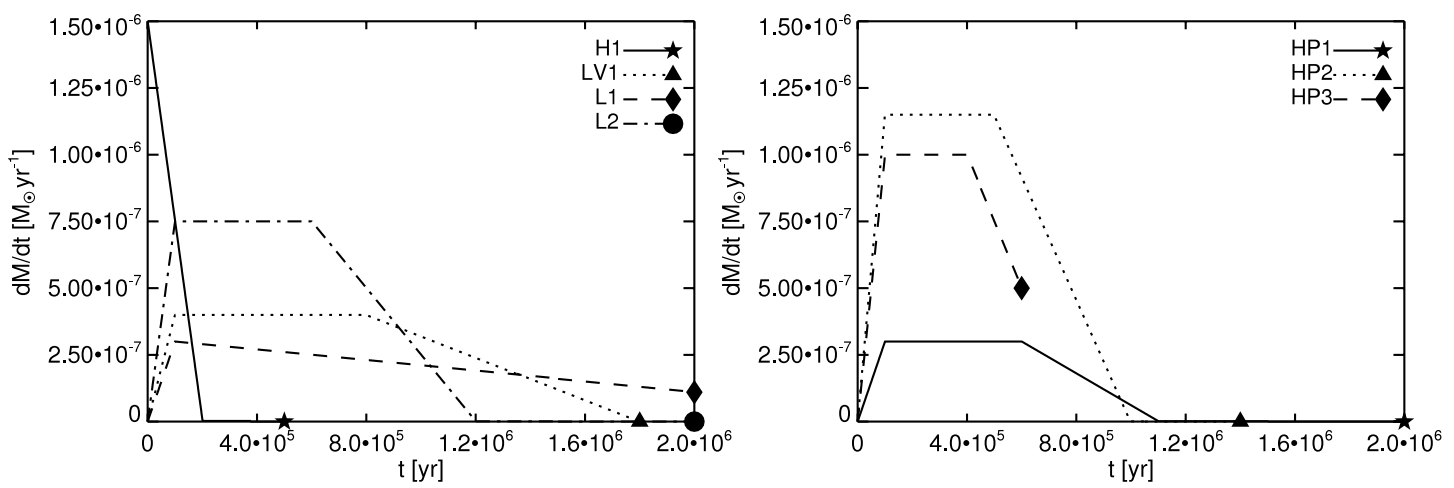

FIg. 1.- Temporal evolution of the mass-loss rates in the outflow models for Type Ia SN progenitor systems detailed in Table 1. Left: Models H1, LV1, L1, and L2. Right: Models HP1, HP2, and HP3. The symbols mark the time of the SN explosion, $t_{\mathrm{SN}}$.

(§3.3), and then we examine the consequences of varying them $(\S 3.4)$.

\subsection{Theory}

A complete theoretical overview of the interaction between stellar winds and the surrounding medium is given by Koo \& McKee (1992a, 1992b), who built on the pioneering work of Castor et al. (1975) and Weaver et al. (1977). In the framework set by this theory, we can assimilate the accretion wind outflows to stellar winds, and expect them to form cavities or "accretion-wind-blown bubbles" around the Type Ia progenitors, similar to the more familiar stellar-wind-blown bubbles found around the massive progenitors of core-collapse SNe (see Dwarkadas 2005 and references therein). In general, these cavities will have the usual structure of two shocks and a contact discontinuity (CD) separating four regions from the inside out: freely expanding outflow, shocked outflow, shocked ISM, and unperturbed ISM. The detailed configuration and radial extent of the cavity will depend on the temporal evolution of the outflow mechanical luminosity $L_{\text {of }}=(1 / 2) \dot{M}_{\text {of }} u_{\text {of }}^{2}$, the duration of the mass-loss episode, and the pressure exerted by the ISM (for a review of all the possibilities, see $\S 7$ in Koo \& McKee 1992b). One parameter that is fundamental for the evolution of the CSM structure is the critical outflow velocity $v_{\mathrm{cr}}$, given by

$$
v_{\mathrm{cr}}=10^{4}\left(\frac{\dot{M}_{\mathrm{of}} u_{\mathrm{of}}^{2}}{2} \frac{\rho_{\mathrm{ISM}}}{\mu_{\mathrm{H}}}\right)^{1 / 11} \mathrm{~cm} \mathrm{~s}^{-1},
$$

where $\mu_{\mathrm{H}}=2.34 \times 10^{-24} \mathrm{~g}$ is the mean mass per $\mathrm{H}$ atom in a solar abundance gas and all magnitudes are in cgs units (expression adapted from eq. [2.5] in Koo \& McKee 1992a). Outflows with $v_{\text {of }}>v_{\text {cr }}$ are "fast," meaning that radiative losses do not affect the shocked outflow, and the expansion of the outer shock is driven by the thermal energy of the shocked material inside the cavity (energy-driven bubbles). Outflows with $v_{\text {of }}<$ $v_{\text {cr }}$ are "slow," and radiative losses affect the shocked outflow, either at the reverse shock or at the $\mathrm{CD}$. These cavities are driven by the ram pressure of the outflow itself (momentum-driven bubbles). For the values of $\dot{M}_{\text {of }}$ and $\rho_{\text {ISM }}$ typical of accretion winds expanding into the warm ISM, the outflows are in the fast regime at velocities above $\sim 100 \mathrm{~km} \mathrm{~s}^{-1}$ (see Fig. 2).

\subsection{Results: Reference Values}

The structure of the CSM at the time of the SN explosion for each outflow model at the reference values of $v_{\mathrm{of}}, \rho_{\mathrm{ISM}}$, and $T_{\mathrm{ISM}}$ is shown in Figure 3. The fundamental properties of the CSM profiles are summarized in Table 2. As expected for outflows in the fast regime with timescales of $\sim 10^{6} \mathrm{yr}$, all the accretion winds excavate very large low-density cavities around the Type Ia progenitor systems, with radii $R_{c}$ between 17 and $36 \mathrm{pc}$. The temporal evolution of these cavities can be divided into two distinct phases. During the first phase, the outer shock is radiative and the bubble expands at supersonic velocities. Thin shells of radiatively cooled material develop behind the outer shock, and then become thicker as the temperature of the material drops to $10^{2} \mathrm{~K}$ and cooling

TABLE 1

Outflow Models for Type Ia Progenitor Systems

\begin{tabular}{|c|c|c|c|c|c|c|}
\hline \multirow[b]{2}{*}{ Model Name } & \multirow[b]{2}{*}{$\begin{array}{c}M_{\text {of }} \\
\left(M_{\odot}\right)\end{array}$} & \multirow[b]{2}{*}{$\begin{array}{c}t_{\mathrm{SN}} \\
(\mathrm{yr})\end{array}$} & \multicolumn{3}{|c|}{ Binary System Parameters } & \multirow[b]{2}{*}{ REFERENCE } \\
\hline & & & $\begin{array}{c}M_{\mathrm{WD}, 0} \\
\left(M_{\odot}\right)\end{array}$ & $\begin{array}{l}M_{\mathrm{D}, 0} \\
\left(M_{\odot}\right)\end{array}$ & $\begin{array}{c}P_{0} \\
\text { (days) }\end{array}$ & \\
\hline $\mathrm{H} 1$ & 0.15 & $5.0 \times 10^{5}$ & 1.0 & 2.0 & 2.0 & 1 (Fig. 7) \\
\hline 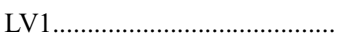 & 0.50 & $1.8 \times 10^{6}$ & 1.0 & 2.5 & 1.6 & 2 (Fig. 1) \\
\hline HP1 . & 0.24 & $2.0 \times 10^{6}$ & 0.75 & 2.0 & 1.58 & 3 (Fig. $1 a$ ) \\
\hline 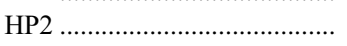 & 0.80 & $1.4 \times 10^{6}$ & 0.8 & 2.2 & 2.50 & 3 (Fig. 1c) \\
\hline 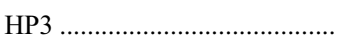 & 0.50 & $6.0 \times 10^{5}$ & 1.0 & 2.4 & 3.98 & 3 (Fig. 1e) \\
\hline 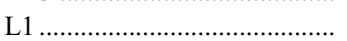 & 0.40 & $2.0 \times 10^{6}$ & 1.0 & 2.3 & 1.74 & 4 (model 2, Fig. 7 ) \\
\hline L2 & 0.64 & $2.0 \times 10^{6}$ & 0.8 & 2.1 & 1.53 & 4,5 (model 31, Fig. 36 in ref. 5 ) \\
\hline
\end{tabular}

Notes.-Outflow model parameters: $M_{\mathrm{of}}$, total mass lost by the system; $t_{\mathrm{SN}}$, time between the onset of the final mass transfer episode and the $\mathrm{SN}$ explosion. Binary system parameters: $M_{\mathrm{WD}, 0}$, initial mass of the WD; $M_{D, 0}$, initial mass of the donor; $P_{0}$, initial period.

ReFERENCES.-(1) Hachisu et al. 1999b; (2) Li \& van den Heuvel 1997; (3) Han \& Podsiadlowski 2004; (4) Langer et al. 2000; (5) Deutschmann 1998. 


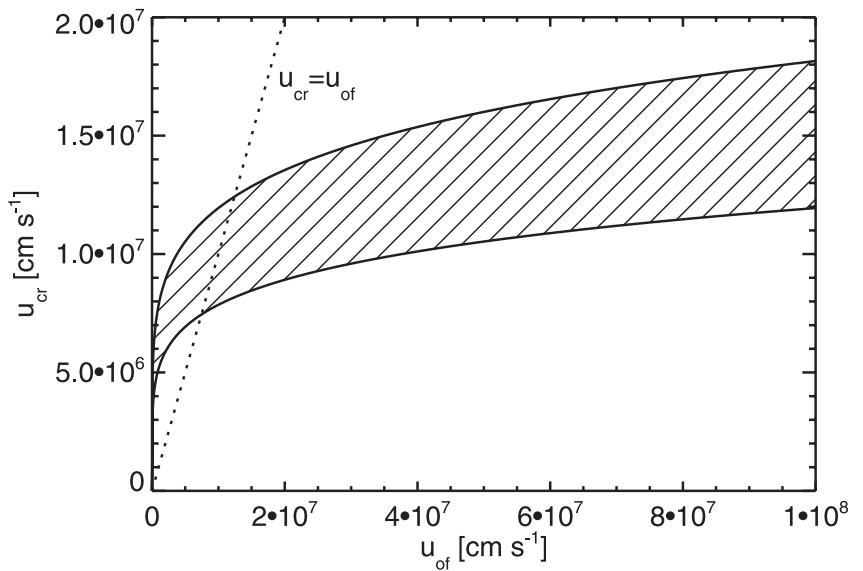

FIG. 2.-Critical outflow velocity $v_{\mathrm{cr}}$ as a function of outflow velocity $v_{\mathrm{of}}$. The striped region represents the values of $v_{\mathrm{cr}}$ for typical accretion wind outflows expanding into the warm, atomic phase of the ISM. The limiting curves are for $\dot{M}_{\mathrm{of}}=$ $10^{-6} M_{\odot} \mathrm{yr}^{-1}, \rho_{\text {ISM }}=5 \times 10^{-24} \mathrm{~g} \mathrm{~cm}^{-3}$ (top curve) and $\dot{M}_{\text {of }}=10^{-7} M_{\odot} \mathrm{yr}^{-1}$, $\rho_{\text {ISM }}=5 \times 10^{-25} \mathrm{~g} \mathrm{~cm}^{-3}$ (bottom curve). The dotted line has a slope of 1 .

ceases. In the terminology introduced by Koo \& McKee (1992b), this is an adiabatic bubble with a radiative outer shock (ABROS), but the only accretion-wind-blown cavity that is still in this stage at $t_{\mathrm{SN}}$ is that generated in model HP3. Eventually, the pressure inside the cavity drops below the ISM pressure (which is $p_{\text {ISM }}=$

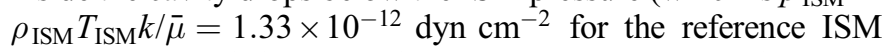
parameters), and the cavity becomes pressure-confined. During this stage, the outer shock becomes a standard sound wave that rapidly dissipates, and the bubble expands (or contracts) at subsonic speeds (adiabatic pressure-confined bubble [APCB]; see $\S 4.4$ in Koo \& McKee 1992b). Models H1, LV1, L1, L2, HP1, and HP2 are in this stage at $t_{\mathrm{SN}}$. The reverse shock can only be seen in the two models that have an active outflow at $t_{\mathrm{SN}}$ (HP3 and L1). In these cases, the progenitor system is surrounded by a small region of unshocked outflow with mass $M_{\text {uof }}$ that extends to a radius $R_{\mathrm{uof}}$.

\subsection{Results: Modified Values}

Among the seven outflow models presented in Table 1, we have chosen models HP3 and L2 as representative examples of outflows producing "small" and "large" cavities. Based on these models, we have explored the range of CSM structures that might be found around Type Ia progenitors by modifying the properties of the ISM and the values of $v_{\text {of }}$.

Variation of the ISM properties.-The reference values of $\rho_{\text {ISM }}$ and $T_{\text {ISM }}$ are at the high end of the expected ranges for the warm atomic phase of the ISM (Ferrière 2001). This choice maximizes the value of $p_{\text {ISM }}$, so that the pressure containment of the wind-blown cavities is maximum, and the size of the wind-blown bubbles shown in Figure 3 is a lower limit. To evaluate the effect of decreasing $p_{\text {ISM }}$ to the lowest reasonable value for the warm ISM, we have evolved the outflow models HP3 and L2 into an ISM with $\rho_{\text {ISM }}=5 \times 10^{-25} \mathrm{~g} \mathrm{~cm}^{-3}$ and $T_{\text {ISM }}=5 \times 10^{3} \mathrm{~K}$. This translates into an ISM pressure of $p_{\text {ISM }}=3.33 \times 10^{-13} \mathrm{dyn}^{-2}$, roughly an order of magnitude below the reference value. The results can be seen in Table 2 and Figure 4, labeled as models HP3lowp and L2lowp. These bubbles are 20\% larger than the reference cases, but there are no significant differences in the structure of the cavities, because the outflows stay in the fast regime. Even at the lower value of $p_{\text {ISM }}$, model L2lowp is already making the transition to the APCB stage at $t_{\mathrm{SN}}$.

Variation of $v_{\text {of }}$ - - We have calculated the CSM structures obtained by models HP3 and L2 with outflow velocities of $10 \mathrm{~km} \mathrm{~s}^{-1}$
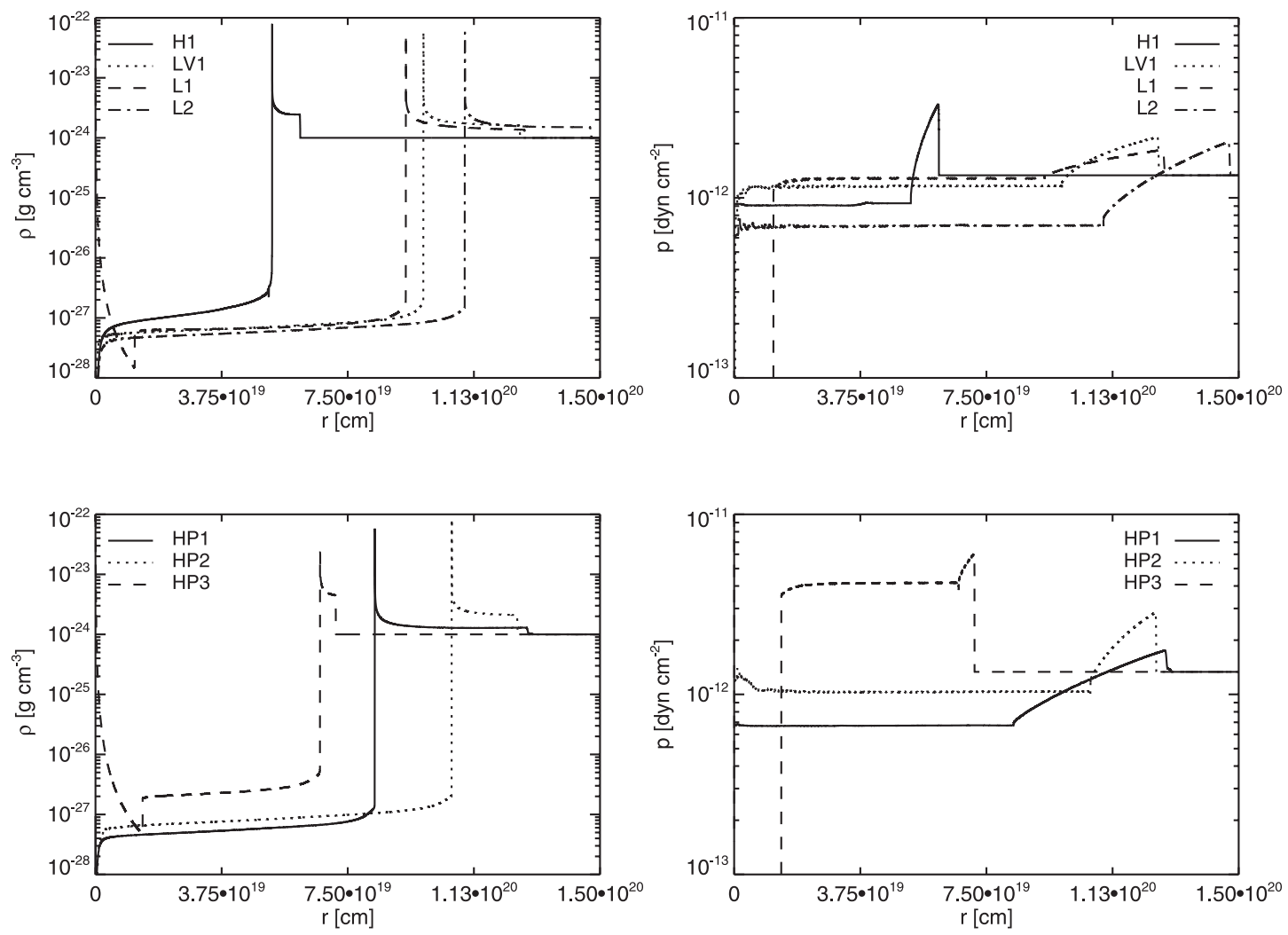

FIG. 3. - Radial structure of the CSM sculpted by the outflow models with the reference values $v_{\text {of }}=10^{3} \mathrm{~km} \mathrm{~s}^{-1}, \rho_{\text {ISM }}=10^{-24} \mathrm{~g} \mathrm{~cm}^{-3}$, and $T_{\text {ISM }}=10^{4} \mathrm{~K}$. Density is shown in the left panels, and pressure is shown in the right panels. 
TABLE 2

Structure of the Cavities Produced by Type Ia Progenitor Outflows

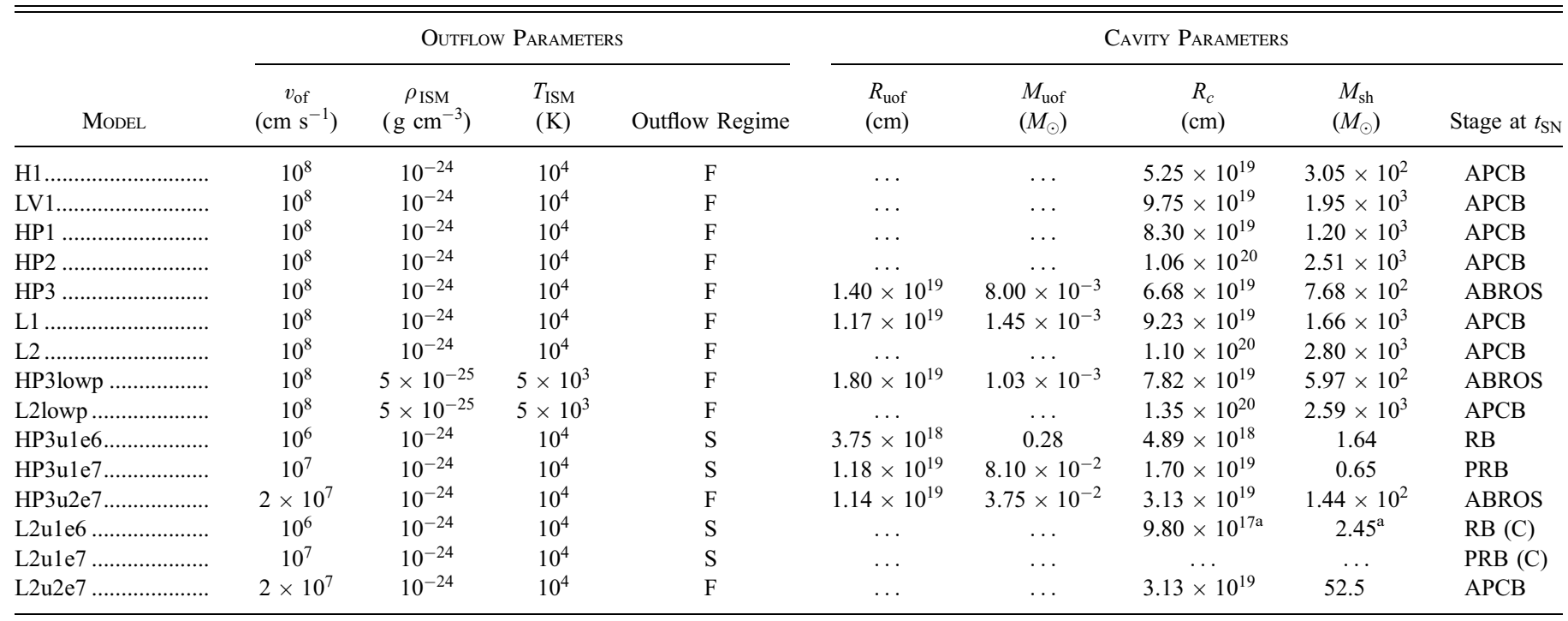

Notes.-Outflow regime: fast (F) or slow (S). Cavity parameters: $R_{\text {uof }}$, radius of the unshocked outflow; $M_{\text {uof }}$, mass of the unshocked outflow; $R_{c}$, radius of the CD (for models in the fast outflow regime, this is equal to the inner radius of the low-density cavity); $M_{\mathrm{sh}}$, mass in the radiatively cooled shell (for cavities that are not pressure confined, $M_{\mathrm{sh}}$ is the mass contained between $R_{c}$ and the outer supersonic blast wave). For the cavity stage at $t_{\mathrm{SN}}$, we use the terminology of Koo \& McKee (1992b). APCB, adiabatic pressure-confined bubble; ABROS, adiabatic bubble with a radiative outer shock; RB, radiative bubble; PRB, partially radiative bubble. Cavities marked with "(C)" have collapsed at $t_{\mathrm{SN}}$.

${ }^{a}$ Parameters for the relic shell of the collapsed bubble.

(a reasonable lower limit, being the typical value for the sound speed in the ISM), $100 \mathrm{~km} \mathrm{~s}^{-1}$, and $200 \mathrm{~km} \mathrm{~s}^{-1}$. The results of these calculations are presented in Table 2 and Figure 5 (models Hp3u1e6, Hp3u1e7, Hp3u2e7, L2u1e6, L2u1e7, and L2u2e7). The lowest values of $v_{\text {of }}$ in this sequence $\left(10\right.$ and $\left.100 \mathrm{~km} \mathrm{~s}^{-1}\right)$ lead to outflows in the slow regime and momentum-driven cavities with structures that are very different from the ones we have seen up to now. The types of cavities described by Koo \& McKee (1992a) are nicely laid out by the sequence of HP3 models with increasing values of $v_{\text {of }}$. Model HP $3 \mathrm{u} 1 \mathrm{e} 6$ is a radiative bubble (RB), with a wind shock that is still radiative at $t_{\mathrm{SN}}$; model HP3ule7 is a partially radiative bubble (PRB), with an adiabatic wind shock but active cooling of the shocked outflow at the CD; model HP3u2e7 is already in the fast regime and has become pressure confined at $t_{\mathrm{SN}}(\mathrm{APCB})$. The sequence of L2 models reveals an interesting effect associated with accretion winds that have a mass-conservative phase prior to the $\mathrm{SN}$ explosion. If these outflows are in the fast regime, the energy-driven cavity survives for a long time, although it may become pressure confined and start to shrink (like model L2u2e7, which is an APCB at $t_{\mathrm{SN}}$ ). If the outflow is in the slow regime, on the other hand, the momentumdriven cavities collapse when the accretion wind ceases. In the case of model L2ule6, which is a RB during the active outflow phase, a relic shell of cooled material is left behind, but the PRB of model L2u1e7 (which looked very similar to HP3u1e7 in the active phase) has disappeared almost completely at $t_{\mathrm{SN}}$.

We conclude this section with a brief reference to our previous results from $\mathrm{BB} 01$. The hydrodynamic simulations in that paper were very simplified and did not include radiative losses. The cavity sizes for the outflows in the fast regime (models A and C in BB01) are qualitatively correct, but the results for models with outflow velocities of $20 \mathrm{~km} \mathrm{~s}^{-1}$ (models B and D), which are in the slow regime, are incorrect (the simulations were later repeated with the inclusion of radiative losses in Badenes 2004). The main conclusion of BB01 - that fast accretion wind outflows lead to large cavities around Type Ia progenitors - still holds, but the more detailed
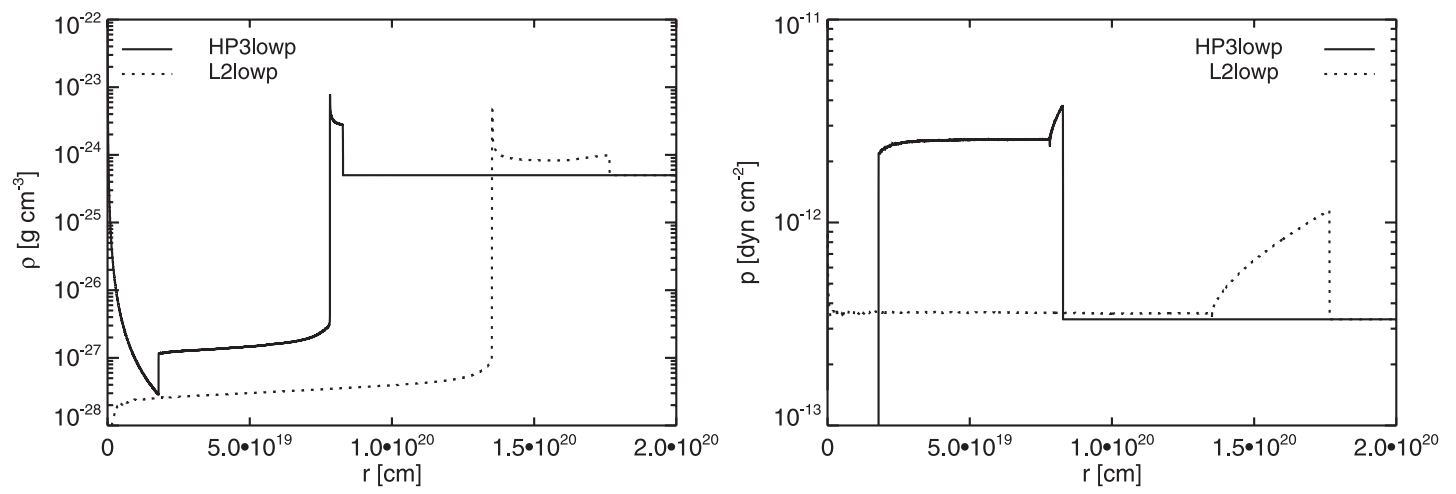

FIG. 4. - Radial structure of the CSM sculpted by the outflow models HP3 and L2 with $\rho_{\text {ISM }}=5 \times 10^{-25} \mathrm{~g} \mathrm{~cm}^{-3}$ and $T_{\text {ISM }}=5 \times 10^{3} \mathrm{~K}$. Density is shown in the left panel, and pressure is shown in the right panel. 

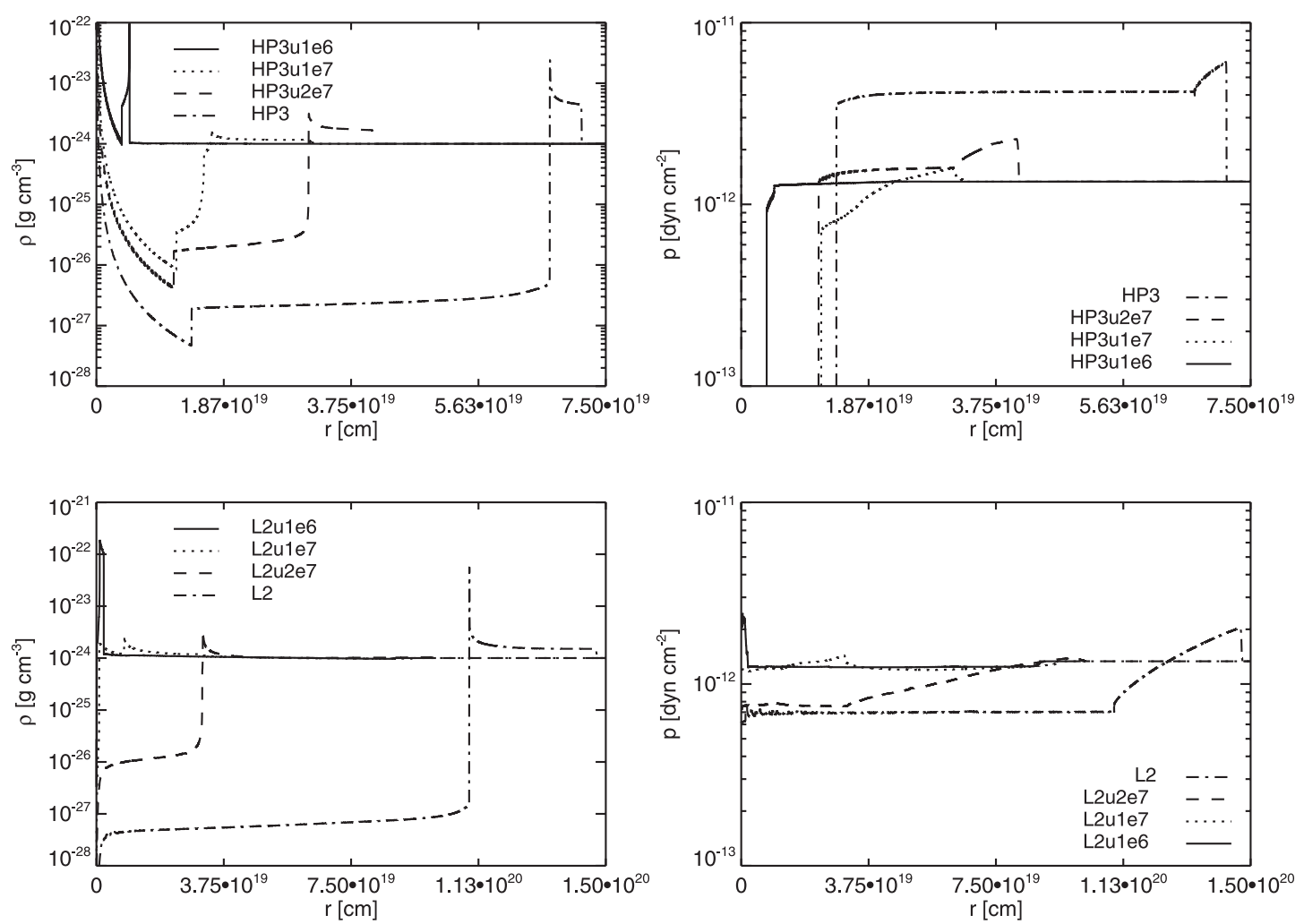

FIG. 5.- Radial structure of the CSM sculpted by the outflow models HP3 (top panels) and L2 (bottom panels), with $v_{\text {of }}=10 \mathrm{~km} \mathrm{~s}^{-1}\left(\right.$ solid lines), $v_{\text {of }}=10^{2} \mathrm{~km} \mathrm{~s}^{-1}$ (dotted lines), $v_{\mathrm{of}}=2 \times 10^{2} \mathrm{~km} \mathrm{~s}^{-1}$ (dashed lines), and $v_{\mathrm{of}}=10^{3} \mathrm{~km} \mathrm{~s}^{-1}$ (dash-dotted lines; the same as in Fig. 3, added for comparison). Density is shown in the left panels, and pressure is shown in the right panels.

results that we present here supersede the calculations in our previous work.

\section{SNR DYNAMICS IN THE MODIFIED CIRCUMSTELLAR MEDIUM}

The dynamics of SNRs expanding into low-density windblown cavities has been studied by several authors (Tenorio-Tagle et al. 1990, 1991; Dwarkadas 2005). The reader is encouraged to consult these works for discussions; we will not go into the details here. Essentially, the SN ejecta expand almost freely until the inner edge of the cavity $R_{c}$ is encountered. What happens after that depends on the momentum of the ejecta and the mass that is contained in the shell that surrounds the cavity, $M_{\mathrm{sh}}$. Dwarkadas (2005) described this interaction in terms of the $\Lambda$-parameter, defined as the quotient between the shell mass and the ejecta mass, $\Lambda=M_{\mathrm{sh}} / M_{\mathrm{ej}}$. Among the CSM structures presented in $\S 3$, those generated by outflows in the fast regime always correspond to the more extreme $\Lambda \gg 1$ scenario (see the values of $M_{\text {sh }}$ listed in Table 2). In this case, the forward shock (FS) becomes radiative when it hits the shell, and the Sedov stage (i.e., $R_{\mathrm{FS}} \propto t^{0.4}$ ) is absent from the SNR evolution (see $\S 7.3$ in Dwarkadas 2005). This strong modification of the dynamics will have two important observational consequences. First, the fundamental properties of the FS (radius, velocity, and expansion parameter) at a given SNR age will be very different from the case of an interaction with a uniform ISM. Second, since most of the ejecta will expand to very low densities before being overrun by the reverse shock, the X-ray emission from the shocked ejecta in the SNR will also be affected, leading to a much lower ionization state at a given age. The CSM structures generated by outflows in the slow regime are more diverse, and their imprint on the FS properties and the X-ray emission from the shocked ejecta will not be so dramatic.
In order to produce SNR models that can be compared with the observations, we have used VH-1 to simulate the interaction of the Type Ia explosion model PDDe from Badenes et al. (2003) with several of the CSM profiles presented in $\S 3$. The choice of a particular Type Ia SN explosion model does not have a significant influence on the radius and velocity of the FS, as long as the kinetic energy produced in the explosion is $\sim 10^{51}$ erg (see Figs. $3 a$ and $3 c$ in Badenes et al. 2003). The X-ray emission from the shocked ejecta, on the other hand, is profoundly affected by the differences in density and chemical composition profiles of Type Ia SN explosion models. We have chosen model PDDe because it maximizes the ionization state of the elements in the shocked ejecta for a given value of $\rho_{\text {ISM }}$ (compare panel $6 d$ with the other panels of Fig. 6 in Badenes et al. 2003). We have followed the nonequilibrium ionization processes in the shocked ejecta with the ionization code described in Badenes et al. (2003), with one important modification. Some extreme cases of CSM interaction that we will specify in $\S 5.3$ lead to catastrophic cooling in the outer ejecta layers. To model this process, we have included ionization and radiative losses in the code, using the latest atomic data published by the CHIANTI collaboration (Dere et al. 1997; Landi et al. 2006). To avoid recalculating the hydrodynamic evolution in each case, we have assumed isobaric cooling in the affected layers (i.e., the layers cool but remain in hydrostatic equilibrium with their surroundings), which is an excellent approximation for the intershock region in one-dimensional hydrodynamics.

\section{COMPARISON WITH OBSERVATIONS}

\subsection{Observations: Forward-Shock Dynamics and X-Ray Emission from the Shocked Ejecta}

We have compiled the relevant observational data for young SNRs that have a firm Type Ia identification in Tables 3 and 4. 
TABLE 3

Type Ia Supernova Remnants: Forward-Shock Dynamics

\begin{tabular}{|c|c|c|c|c|c|}
\hline Remnant Name & $\begin{array}{l}\text { Age } \\
(\mathrm{yr})\end{array}$ & $\begin{array}{c}D \\
(\mathrm{kpc})\end{array}$ & $\begin{array}{c}\alpha_{\mathrm{FS}} \\
(\operatorname{arcmin})\end{array}$ & $\begin{array}{l}R_{\mathrm{FS}} \\
(\mathrm{cm})\end{array}$ & $\begin{array}{c}v_{\mathrm{FS}} \\
\left(\mathrm{cm} \mathrm{s}^{-1}\right)\end{array}$ \\
\hline SN $1885 \ldots \ldots \ldots \ldots \ldots \ldots$ & $121^{\mathrm{a}}$ & $785 \pm 30^{\mathrm{b}}(1)$ & $6.7 \times 10^{-3}(1)$ & $(4.7 \pm 0.2) \times 10^{18}$ & \\
\hline 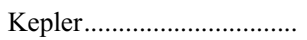 & $402^{\mathrm{a}}$ & $4.8 \pm 1.4(2)$ & 1.7 & $(7.3 \pm 2.1) \times 10^{18}$ & $2.0 \times 10^{8}-2.5 \times 10^{8}(3)$ \\
\hline 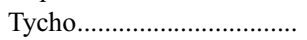 & $434^{\mathrm{a}}$ & $1.5-3.1(4)$ & 4.0 & $(8.3 \pm 2.9) \times 10^{18}$ & $1.9 \times 10^{8}-2.3 \times 10^{8}(5)$ \\
\hline 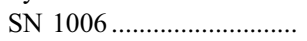 & $1000^{\mathrm{a}}$ & $1.4-2.8^{\mathrm{c}}(4)$ & 15.0 & $(2.8 \pm 0.9) \times 10^{19}$ & $2.8 \times 10^{8}-3.0 \times 10^{8}(3)$ \\
\hline 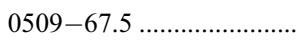 & $400 \pm 120^{\mathrm{d}}(6)$ & $50 \pm 1^{\mathrm{e}}(7)$ & $0.24^{\mathrm{f}}$ & $1.1 \times 10^{19}$ & $>2.0 \times 10^{8}(5)$ \\
\hline 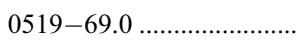 & $600 \pm 200^{\mathrm{d}}(6)$ & $50 \pm 1^{\mathrm{e}}(7)$ & $0.26^{\mathrm{f}}$ & $1.2 \times 10^{19}$ & $1.0 \times 10^{8}-1.9 \times 10^{8}(5)$ \\
\hline 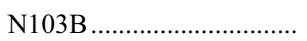 & $860 \pm 400^{\mathrm{d}, \mathrm{g}}(6)$ & $50 \pm 1^{\mathrm{e}}(7)$ & $0.23^{\mathrm{f}}$ & $1.0 \times 10^{19}$ & $\ldots$ \\
\hline
\end{tabular}

Notes.-SNR parameters: $D$, distance to the $\mathrm{SNR} ; \alpha_{\mathrm{FS}}$, angular radius of the $\mathrm{FS} ; R_{\mathrm{FS}}, \mathrm{FS}$ radius $\left(R_{\mathrm{FS}}=3.09 \times 10^{21} D\left(\alpha_{\mathrm{FS}} / 60\right)(\pi / 180)\right) ; v_{\mathrm{FS}}, \mathrm{FS}$ velocity.

${ }^{\text {a }}$ Historical SNR.

b Distance to M31.

${ }^{c}$ For the distance to SN 1006, we use the more conservative estimate of Smith et al. (1991), which includes systematic as well as statistical uncertainties, instead of the newer results of Winkler et al. (2003).

${ }^{\mathrm{d}}$ Age estimate from light echoes.

e Distance to the LMC.

${ }^{\mathrm{f}}$ For the LMC SNRs, the radii have been determined by the outermost extent of the X-ray emission in the $0.5-4 \mathrm{keV}$ band, subtracting $0.5^{\prime \prime}$ due to the smearing of the rim by the Chandra PSF.

${ }_{\mathrm{g}}$ The uncertainty in the age of N103B arbitrarily set to $400 \mathrm{yr}$. Rest et al. (2005) determined an age of $860 \mathrm{yr}$, but could not constrain the uncertainty in the measurement.

References.-(1) Fesen et al. 2007; (2) Reynoso \& Goss 1999; (3) Sollerman et al. 2003; (4) Smith et al. 1991; (5) Ghavamian et al. 2001; (6) Rest et al. 2005; (7) Alves 2004.

We have restricted ourselves to objects that are close enough to study the dynamics of the FS and (if possible) the X-ray emission from the shocked ejecta, and whose age is either known (i.e., historical SNRs) or can be estimated with a high degree of confidence. This yields seven objects: SN 1885 in M31; Tycho, Kepler, and SN 1006 in our Galaxy; and 0509-67.5, 0519-69.0, and N103B in the Large Magellanic Cloud (LMC). Among these objects, SN 1885 was identified as a Type Ia SN from the optical spectrum recorded in the nineteenth century (de Vaucouleurs \& Corwin 1985), although it was probably a subluminous event (Chevalier \& Plait 1988; Fesen et al. 2007). Tycho and SN 1006 have been traditionally regarded as prototypical Type Ia SNRs based on their X-ray spectra and other evidence, and their origin has been confirmed by detailed modeling of the ejecta emission (for Tycho, see Badenes et al. 2006; for SN 1006, see C. Badenes et al. 2007, in preparation). The only object in our sample whose origin is somewhat controversial is the Kepler SNR (for a discussion and references, see Cassam-Chenai et al. 2004), but the prominent $\mathrm{Fe}$ emission and the virtual absence of $\mathrm{O}$ in the shocked ejecta revealed by the recent deep Chandra observations (Reynolds et al. 2007) are strongly indicative of a Type Ia origin. The LMC SNRs 0509-67.5, 0519-69.0, and N103B were first identified as

TABLE 4

Type Ia Supernova Remnants: Ionization Timescales in the Shocked EJecta

\begin{tabular}{|c|c|c|}
\hline \multirow[b]{2}{*}{ Remnant Name } & \multicolumn{2}{|c|}{$\log \left(\left\langle n_{e} t\right\rangle\right)\left(\mathrm{cm}^{-3} \mathrm{~s}\right)$} \\
\hline & $\mathrm{Si}$ & $\mathrm{Fe}$ \\
\hline 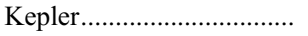 & $10.08-10.24$ & $9.85-9.92$ \\
\hline Tycho & $10.23-10.99$ & $9.72-9.78$ \\
\hline SN 1006 & $9.49-9.60$ & $\ldots{ }^{a}$ \\
\hline $0509-67.5 \ldots \ldots \ldots \ldots \ldots \ldots \ldots . .$. & $9.80-9.82$ & $<9.7$ \\
\hline $0519-69.0 \ldots \ldots \ldots \ldots \ldots \ldots . .$. & $10.50-11.62$ & $9.90-9.95$ \\
\hline N103B & $10.64-11.94$ & $10.62-10.69$ \\
\hline
\end{tabular}

${ }^{\text {a }} \mathrm{SN} 1006$ has no Fe K emission in its Chandra X-ray spectrum (J. P. Hughes et al. 2007, in preparation).
Type Ia by Hughes et al. (1995). The results of later works appear to agree with the Type Ia hypothesis in the cases of 0509-67.5 (Warren \& Hughes 2004) and N103B (Lewis et al. 2003), but there are no detailed models in the literature that can confirm this. An interesting development for these LMC SNRs has been the recent detection of light echoes from the explosion, which has greatly reduced the uncertainty in their ages and might eventually confirm their Type Ia origin (Rest et al. 2005).

The radius and velocity of the FS in a SNR provide a first assessment of its dynamical state. The FS radii $R_{\mathrm{FS}}$ are determined from the angular radii $\alpha_{\mathrm{FS}}$ and the distances to the $\mathrm{SNRs} D\left(R_{\mathrm{FS}}=\right.$ $\alpha_{\mathrm{FS}} D$ ). All the angular radii listed in Table 3 are from Chandra observations except that of SN 1885, which is from the absorption features seen in HST images (Fesen et al. 2007). The X-ray radii are very precise: even in the LMC SNRs, the Chandra PSF will only introduce errors below $\sim 5 \%$. The value of $D$ is known with some accuracy for the LMC SNRs (Alves 2004) and for SN 1885 (Fesen et al. 2007), but it can be very uncertain in the Galactic SNRs. The FS velocities are obtained from the study of the narrow and broad components of the $\mathrm{H} \alpha$ emission in nonradiative shocks (Chevalier \& Raymond 1978). The derivation of $v_{\mathrm{FS}}$ from the width of the broad $\mathrm{H} \alpha$ component is not straightforward, and it requires some modeling of the plasma physics at the shock. It is important to note that the FS velocities are obtained at specific points in the blast wave (usually those that offer the best signal-to-noise ratio in $\mathrm{H} \alpha$ ), and may not be representative of the dynamics of the entire SNR. Furthermore, Heng \& McCray (2007) recently expressed concern that the current models might be underestimating the shock velocities from the $\mathrm{H} \alpha$ observations. For these reasons, the values of $v_{\mathrm{FS}}$ listed in Table 3 need to be considered with caution.

We have used the average ionization timescale $\left\langle n_{e} t\right\rangle$ of $\mathrm{Fe}$ and $\mathrm{Si}$ to represent the ionization state of these elements in the shocked ejecta of our SNRs. In order to assemble a consistent set of values and constrain the uncertainties in the best possible way, we have determined the ionization timescales listed in Table 4 from archival Chandra observations of our SNR sample. The methods and techniques that we have used for this purpose are detailed in the Appendix. 

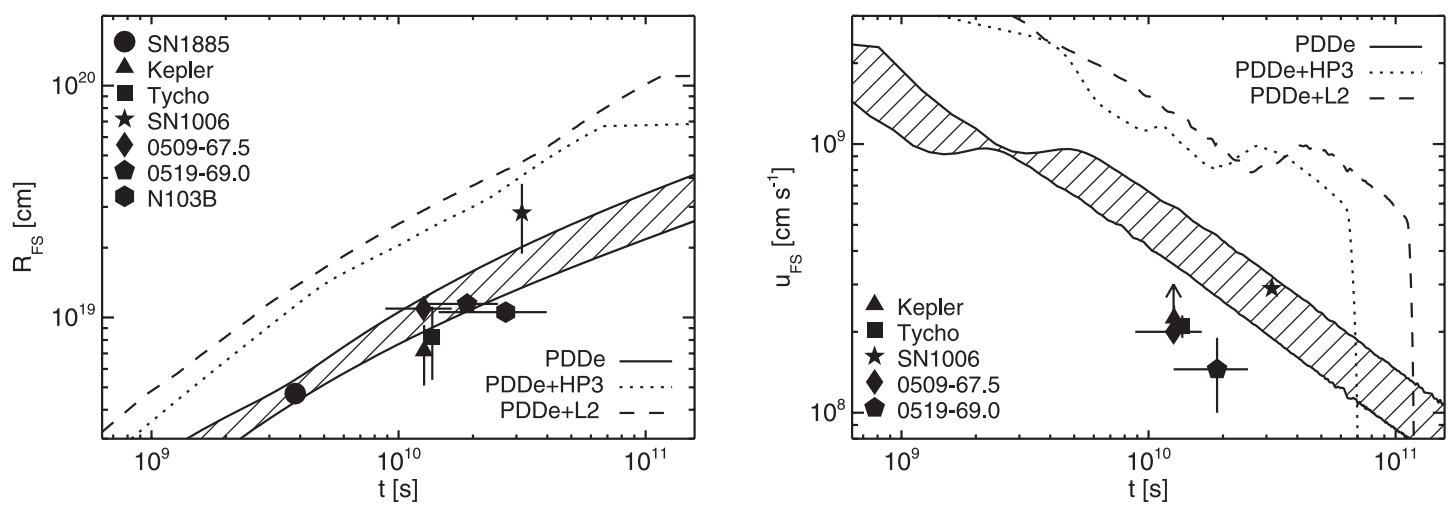

FIG. 6.-Temporal evolution of the forward-shock radius $R_{\mathrm{FS}}(l e f t)$ and forward-shock velocity $v_{\mathrm{FS}}(r i g h t)$ for Type Ia SN explosion model PDDe interacting with a constant-density ISM (solid lines) and with the CSM profiles HP3 (dotted line) and L2 (dashed line). The two solid lines correspond to an interaction with $\rho_{\text {ISM }}=$ $5 \times 10^{-25} \mathrm{~g} \mathrm{~cm}^{-3}$ (top lines) and $\rho_{\text {ISM }}=5 \times 10^{-24} \mathrm{~g} \mathrm{~cm}^{-3}$ (bottom lines). The striped region between the solid lines spans the parameter space of $\rho_{\text {ISM }}$ between these two values. The data from Table 4 are overlaid on the plots.

\subsection{Forward-Shock Dynamics: Models versus Observations}

Before attempting a comparison between the dynamics of the FS in our SNR models and the observed values, we must emphasize that our calculations do not include the effect of efficient shock acceleration of cosmic rays. This process is known to take part in many young SNRs, and recent theoretical (Ellison et al. 2004) and observational (Decourchelle et al. 2000; Warren et al. 2005) works have shown that it can have a noticeable impact on the internal structure and X-ray emission of the SNR, specially in the shocked ISM. The impact on the dynamics of the FS, however, is modest: at an age of $5000 \mathrm{yr}$, the values of $R_{\mathrm{FS}}$ and $v_{\mathrm{FS}}$ in a SNR that is accelerating cosmic rays efficiently are only $\sim 15 \%$ lower than in a test particle case (see Fig. 3 in Ellison et al. 2004). This kind of deviation is inconsequential for the order-ofmagnitude comparisons that we carry out in the present section.

The values of $R_{\mathrm{FS}}$ and $v_{\mathrm{FS}}$ in our SNR models are plotted alongside the observations in Figures 6 and 7. In Figure 6 we display the values obtained for the interaction between the explosion model PDDe and the CSM profiles HP3 and L2 from Figure 3, together with a set of SNR models generated with a uniform ISM. For the uniform ISM interaction, we have represented the parameter space obtained by varying $\rho_{\text {ISM }}$ as a dashed region between two limiting cases, $\rho_{\mathrm{ISM}}=5 \times 10^{-25} \mathrm{~g} \mathrm{~cm}^{-3}$ (top lines) and $\rho_{\mathrm{ISM}}=5 \times$ $10^{-24} \mathrm{~g} \mathrm{~cm}^{-3}$ (bottom lines), which encompass the typical conditions in the warm atomic phase of the ISM. The SNR models
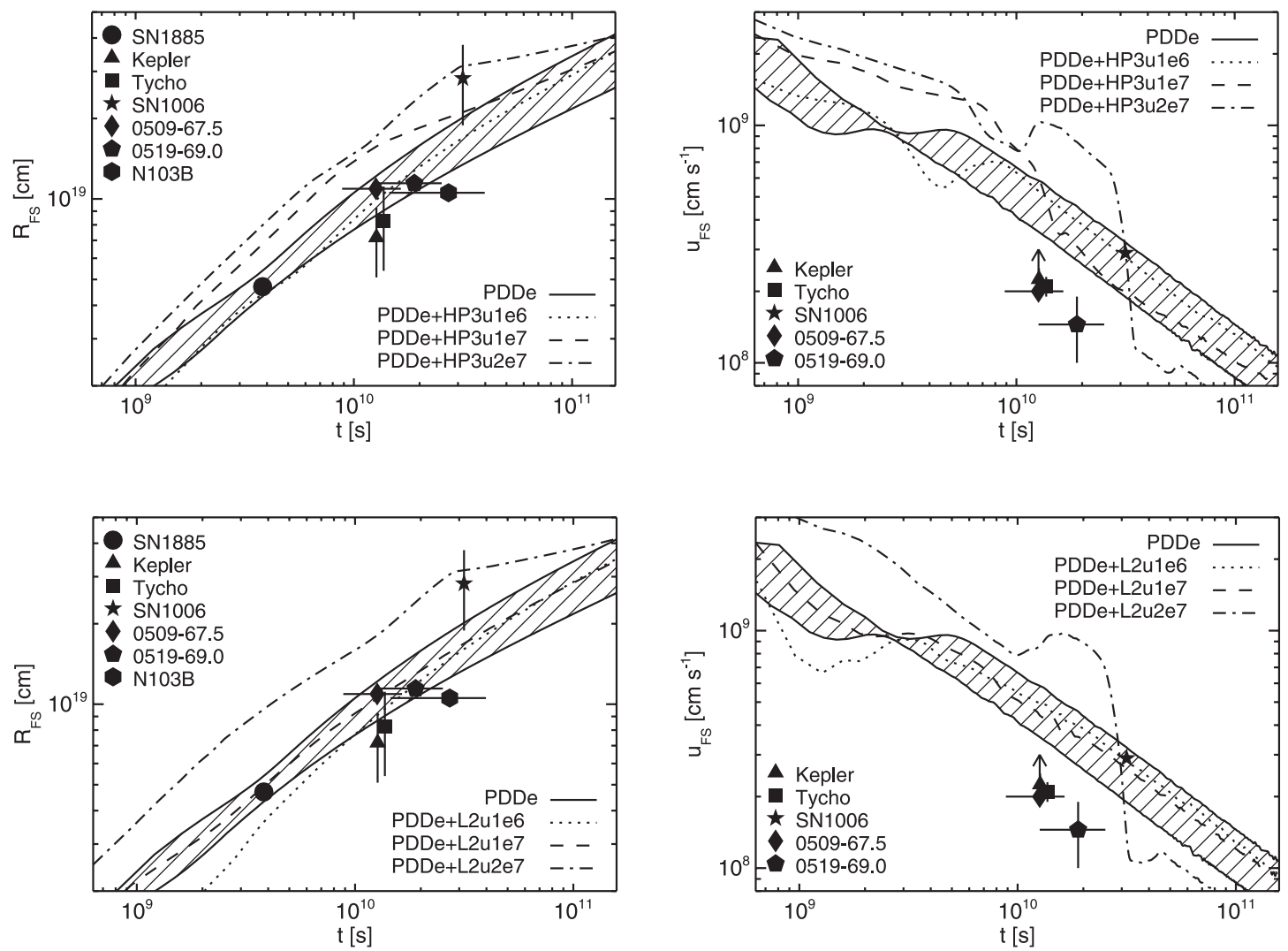

Fig. 7.- Same as Fig. 6, but for the explosion model PDDe interacting with a uniform ISM (solid lines) and the CSM profiles generated by outflows with modified velocities. Top panels: Models HP3ule6 (dotted line), HP3ule7 (dashed line), and HP3u2e7 (dash-dotted line). Bottom panels: Models L2u1e6 (dotted line), L2u1e7 (dashed line), and $\mathrm{L} 2 \mathrm{u} 2 \mathrm{e} 7$ (dash-dotted line). 

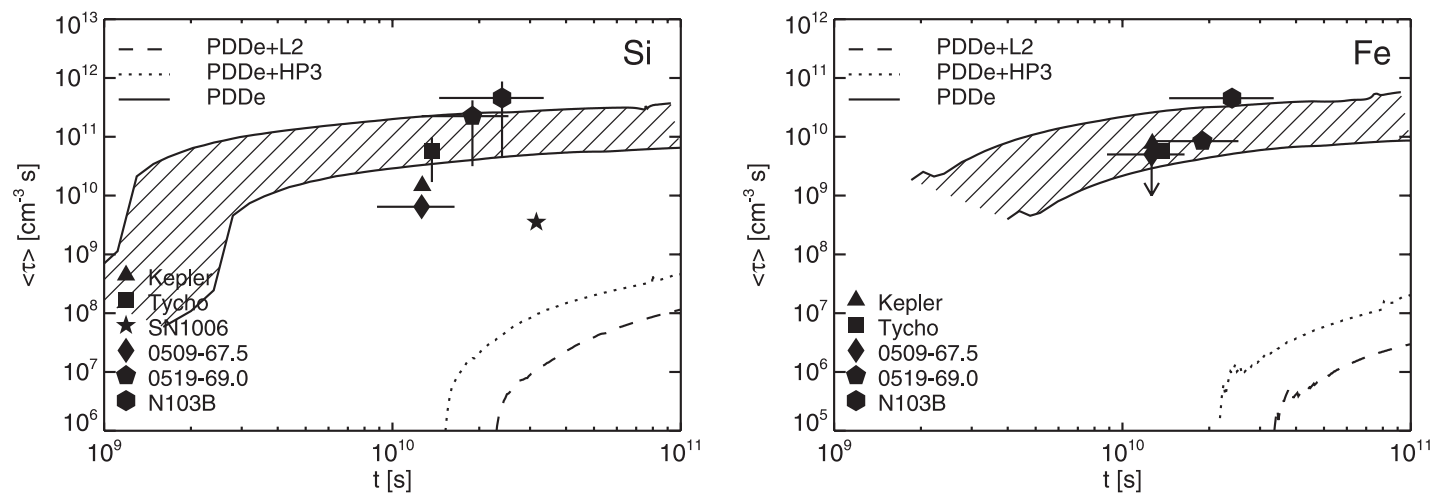

FIG. 8. - Temporal evolution of the emission-measure-averaged ionization timescale $\langle\tau\rangle$ for $\operatorname{Si}($ left $)$ and $\mathrm{Fe}($ right $)$ in the shocked ejecta of model PDDe interacting with a constant-density ISM (solid lines) and with the CSM profiles HP3 (dotted line) and L2 (dashed line). The two solid lines correspond to an interaction with $\rho_{\text {ISM }}=$ $5 \times 10^{-25} \mathrm{~g} \mathrm{~cm}^{-3}$ (top lines) and $\rho_{\text {ISM }}=5 \times 10^{-24} \mathrm{~g} \mathrm{~cm}^{-3}$ (bottom lines). The striped region between the solid lines spans the parameter space of $\rho_{\text {ISM }}$ between these two values. The data from Table 4 are overlaid on the plots.

evolving inside the HP3 and L2 CSM profiles are clearly unable to reproduce the observed values of $R_{\mathrm{FS}}$, but most of the data points cluster nicely around the uniform ISM models. The only exception is SN 1006, whose large size is compatible with both a very low density ISM and the CSM cavity from model HP3. The situation is somewhat different for the FS velocities. While it is clear that the SNR models evolving inside the CSM cavities can be confidently discarded, it seems that the models interacting with a uniform ISM also overpredict the values of $v_{\mathrm{FS}}$, again with the only exception of SN 1006. The distribution of the data points for Kepler, Tycho, 0509-67.5, and 0519-69.0 suggests a systematic shift of a factor $\sim 2$ between the values of $v_{\mathrm{FS}}$ predicted by modeling the $\mathrm{H} \alpha$ emission of the FS and the models with an ISM interaction. This could be the hallmark of the effect discussed by
Heng \& McCray (2007) or a selection effect toward localizations in the FS with higher densities imposed by the statistics of the $\mathrm{H} \alpha$ observations, but more complex explanations cannot be discarded without a detailed analysis.

In Figure 7 we repeat the comparison, this time for SNRs evolving into the cavities obtained with the modified values of $v_{\text {of }}$. From the point of view of the FS dynamics alone, the CSM structures produced by outflows in the slow regime are hard to distinguish from a uniform ISM interaction. This is specially true for the collapsed cavities of models L2u1e6 and L2u1e7 and the small RB of model HP3ule6. The energy-driven cavities of models HP3u2e7 and L2u2e7, on the other hand, are hard to reconcile with the observations of all SNRs except SN 1006. The size of these two cavities is coincidentally similar to the radius of
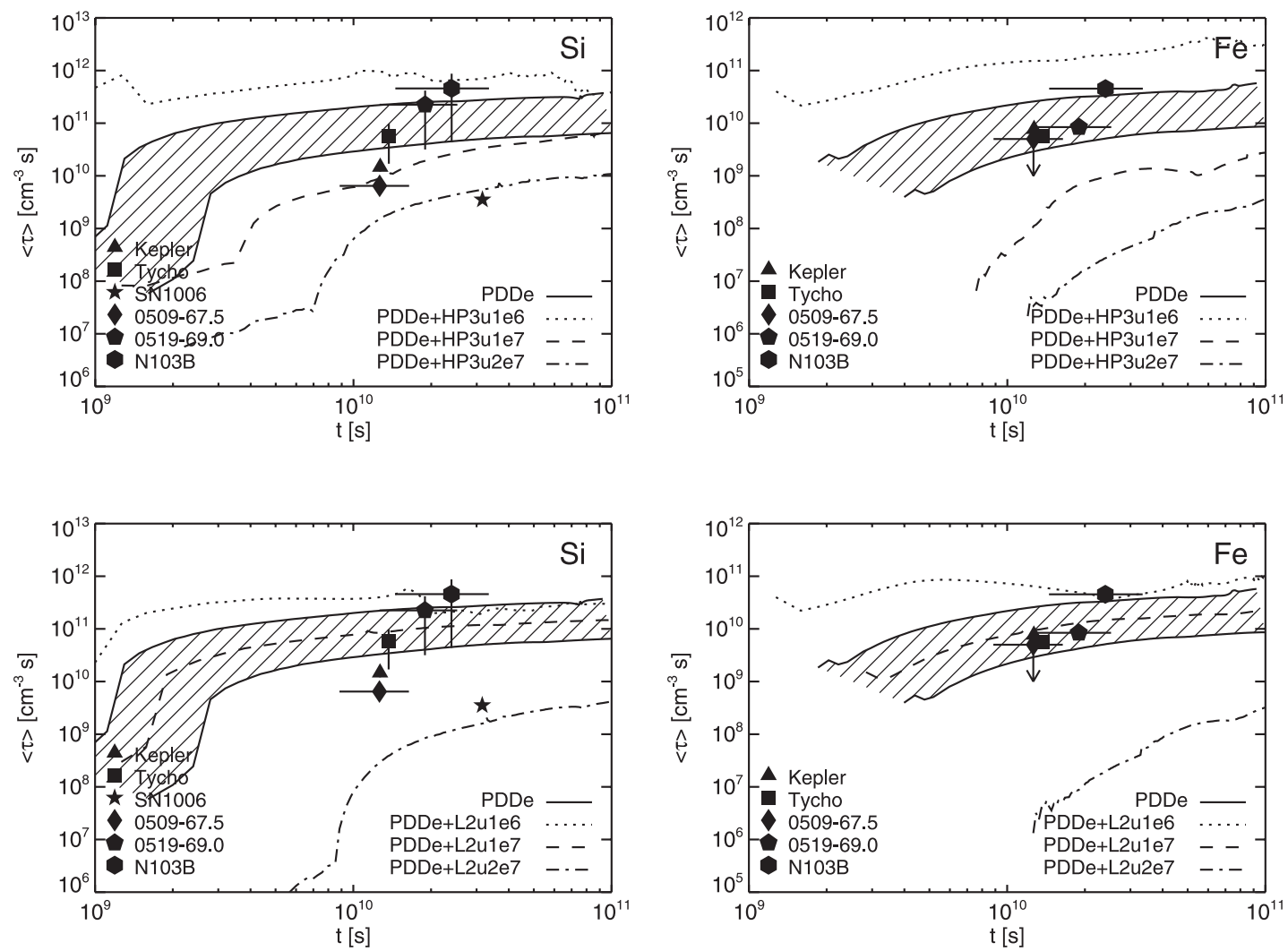

FIG. 9.- Same as Fig. 8, but for the explosion model PDDe interacting with a uniform ISM (solid lines) and the CSM profiles generated by outflows with modified velocities. Top panels: Models HP3u1e6 (dotted line), HP3u1e7 (dashed line), and HP3u2e7 (dash-dotted line). Bottom panels: Models L2u1e6 (dotted line), L2u1e7 (dashed line), and $\mathrm{L} 2 \mathrm{u} 2 \mathrm{e} 7$ (dash-dotted line). 
SN 1006, so that the FS is hitting the shell at $R_{c}$ for a SNR age of $\sim 1000 \mathrm{yr}$. This results in a good match to the present value of $v_{\mathrm{FS}}$ in SN 1006, but in a regime where the FS is undergoing a very rapid deceleration as it overcomes the radiatively cooled shell (Fig. 7, dash-dotted lines). If this was indeed the situation, one would expect to see a radiative FS with a very low expansion parameter in SN 1006, instead of the observed nonradiative FS (Winkler et al. 2003) with an expansion parameter of $\sim 0.48$ (Moffett et al. 1993).

We conclude this section by noting that there is evidence for some kind of CSM interaction in at least two of the objects in our sample, Kepler (Bandiera 1987; Borkowski et al. 1992) and N103B (Lewis et al. 2003). The fact that this is not clearly revealed by the values of $R_{\mathrm{FS}}$ and $v_{\mathrm{FS}}$ listed in Table 3 emphasizes the importance that hydrodynamic models of individual SNRs will have in order to take these comparisons to the next level of detail.

\subsection{X-Ray Emission from the Shocked Ejecta: Models versus Observations}

In Figures 8 and 9, we compare the values of $\left\langle n_{e} t\right\rangle$ for Fe and Si listed in Table 4 to the emission-measure-averaged ionization timescales $(\langle\tau\rangle$ as defined in $\S 4.2$ of Badenes et al. 2003) in our models. These comparisons have to be of a more qualitative sort than those based on the FS dynamics, due both to issues that affect the spectral models that we use to fit the X-ray observations (see Appendix) and to the limitations of our one-dimensional hydrodynamic calculations (see discussion in $\S 8$ of Badenes et al. 2006). In spite of this, we find that qualitative comparisons of ionization timescales are highly informative, and more than sufficient for our present needs.

In Figure 8 we present the temporal evolution of $\langle\tau\rangle$ for $\mathrm{Si}$ and $\mathrm{Fe}$ in the SNR models obtained from the interaction of model PDDe with the CSM profiles HP3 and L2, and with a uniform ISM. For the uniform ISM case, we have represented the variation of $\rho_{\text {ISM }}$ between $\rho_{\text {ISM }}=5 \times 10^{-25}$ and $5 \times 10^{-24}$ as in Figures 6 and 7 . We have scaled $\langle\tau\rangle$ with $\rho_{\text {ISM }}$ using the approximate relations given in $\S 2.3$ of Badenes et al. (2005). Once again, the data points cluster around the models with a uniform ISM interaction, albeit with more dispersion than in the $R_{\mathrm{FS}}$ plots. The inconsistency between the SNR models evolving inside low-density cavities and the observations is even more dramatic than for the FS dynamics. There is simply not enough mass in the CSM models HP3 and L2 that the SN ejecta can react to in order to reach the ionization timescales observed in Type Ia SNRs.

In Figure 9 we repeat the comparison using the CSM profiles obtained with the modified values of $v_{\text {of }}$. As expected, the CSM profiles generated by outflows in the slow regime lead to a stronger interaction with the SN ejecta and higher ionization timescales for $\mathrm{Fe}$ and $\mathrm{Si}$. This is specially true for models HP3ule6 and L2u1e6, which have prominent shells of radiatively cooled material close to the SN progenitor. In these models, some ejecta layers undergo catastrophic cooling early in the evolution of the SNR. Models HP3ule7 and L2ule7 do not lead to such extreme interaction, and are in general hard to distinguish from a uniform ISM. As soon as the outflows enter the fast regime, however, the situation changes radically. Only the shocked ejecta emission of SN 1006, with its low $\mathrm{Si} n_{e} t$ and absence of $\mathrm{Fe}$, is compatible with the CSM cavities HP3u2e 7 and L2u2e7, but these models have already been discarded in $\S 5.2$ for other reasons.

\section{DISCUSSION}

In $\S 3$ we have seen that the accretion wind outflows invoked by current Type Ia progenitor models in the SD channel will excavate large cavities in the ISM. In $\S 5$ we have shown that the fundamental properties of the seven Type Ia SNRs in our sample are incompatible with SNR models that expand into such large cavities. Can these two results be connected with enough confidence to draw conclusions on Type Ia SN progenitor models based on the SNR observations? In this section, we review the potential issues that might affect the initial models, the simulations, and the observational sample, and we briefly discuss the implications of our results for the observations of Type Ia SNe.

\subsection{Initial Models: The True Nature of Accretion Winds}

The outflow models presented in Figure 1 and Table 1 make two important approximations: they are isotropic (i.e., spherically symmetric) in space and continuous in time. In a more realistic scenario, the presence of the donor star and accretion disk and the spin-up of the WD to high angular velocities (Yoon \& Langer 2004, 2005) should introduce some sort of bipolarity in the outflows. As we have seen in $\S 2.2 .2$, the observations of Type Ia SN progenitor candidates show some evidence of this bipolarity. Continuous bipolar outflows may lead to a bipolar CSM structure similar to some planetary nebulae (Balick \& Frank 2002), but this does not seem likely for the objects in our sample. Only moderate one-sided asymmetries are apparent in Kepler and N103B, and most other objects (in particular, Tycho, SN 1006, and 050967.5) are nearly spherical.

Setting aside the spatial structure of the outflows from Type Ia SN progenitors, their temporal evolution will not be as simple as the plots shown in Figure 1. In order to reach the values of $\dot{M}$ that allow for the growth of the WD, the mass transfer rate has to cross the instability region below $\dot{M}_{\text {stable, }}$, where more or less frequent nova outbursts are expected. Langer et al. (2000), for instance, found a long $\left(\sim 10^{6} \mathrm{yr}\right)$ switch-on phase of the mass transfer in all their binary systems that was dominated by instabilities. Nova-like outbursts could also appear during the massconservative phase between the cessation of the accretion wind and the SN explosion (Hachisu et al. 1999b). Judging from the observations of RX J0513.9-6951 and V Sagittae discussed in $\S 2.2 .2$, a steady accretion wind might be impossible or happen only in a few cases. If the episodic outflows in Type Ia progenitors have periodicities of $\sim 100$ days, as in these two systems, several million cycles should take place through the accretion phase, and the outflows might smear out to become indistinguishable from a continuous wind. In the case of recurrent novae, the individual outbursts will be more spaced out, leading to a complex CSM structure, with several shells that brighten up as they are overtaken by the FS. Again, there is no evidence for this in any of the objects that we have studied, but more detailed hydrodynamic calculations would be necessary to confirm or discard any given scenario.

\subsection{Simulations: From Outflows to Cavities}

There are two ways to prevent Type Ia progenitor outflows from leaving large cavities in the CSM: either the value of $v_{\text {of }}$ is decreased below the critical limit $v_{\text {cr }}$ or the cavities are somehow destroyed before the SN explodes. As we have shown in $\S 5$, outflows in the slow regime $\left(v_{\text {of }} \lesssim 100 \mathrm{~km} \mathrm{~s}^{-1}\right)$ lead to CSM structures that are hard to distinguish from a uniform ISM interaction. However, outflows emanating from the WD surface should have much higher velocities, of the order of the escape velocity $\left(\sim 1000 \mathrm{~km} \mathrm{~s}^{-1}\right.$, a value that is supported by the observations cited in $\S 2.2 .2$ ). Some of the kinetic energy of the outflows will be lost in overcoming the potential well of the system, but it is unlikely that this can reduce $v_{\text {of }}$ by an order of 
magnitude. Furthermore, the presence of slow outflows around the progenitor systems is difficult to reconcile with the lack of CSM detections in Type Ia SNe (see $\S 6.4$ ).

If large cavities do form, it is hard to find a noncatastrophic process that could destroy them before $t_{\mathrm{SN}}$. Thermal conduction, for instance, which must be active to some extent at the interface between the hot shocked outflow and the radiatively cooled shell, could not wipe out cavities with a radius of $\sim 10^{20} \mathrm{~cm}$, even over timescales of $\sim 10^{6} \mathrm{yr}$. In the example presented in $\S$ IV of Weaver et al. (1977), which is based on an interstellar bubble very similar to our models, the conduction front behind the cool shell only spans a small fraction of the cavity radius. The density inside the cavity increases due to evaporation of material from the shell, but it remains orders of magnitude below the surrounding ISM.

A final possibility is that the binary system forms a cavity and then leaves it behind as it moves with respect to the ISM. Large speeds are not expected in SD Type Ia progenitors, but even at the mean random velocity of $\sim 20 \mathrm{~km} \mathrm{~s}^{-1}$ (characteristic of the solar neighborhood; Dehnen \& Binney 1998) a star can move $\sim 10^{20} \mathrm{~cm}$ in $2 \times 10^{6} \mathrm{yr}$. Still, only moving systems with an extended mass-conservative phase before the SN explosion could leave their cavities completely behind. In moving progenitors with active outflows at $t_{\mathrm{SN}}$, the explosion would happen off-center, but the cavity would still be there, and therefore the impact on the FS dynamics and the X-ray emission of the SNR would still be strong (Weaver et al. 1977; Rózyczka et al. 1993).

\subsection{Observations: Other Type Ia SNRs?}

We have omitted from our study several SNRs whose X-ray spectra suggest a Type Ia origin, but whose ages can only be roughly estimated from the FS dynamics. Examples include G337.2-0.7 and G299.2-2.9 in the Galaxy (Rakowski et al. 2006; Park et al. 2006), and DEM L71, DEM L238, and DEM L249 in the LMC (Hughes et al. 2003; Borkowski et al. 2006). These are rather old objects, and nothing in their morphology or X-ray emission indicates that they might be expanding inside a large wind-blown cavity, but clues for other kinds of CSM interaction might be revealed by detailed models.

It is also possible that young Type Ia SNRs expanding inside large cavities do exist, but have not been identified yet. These objects would have a large radius and a faint X-ray emission, so they would be hard to classify as Type Ia SNRs. One such object might be the Galactic SNR RCW 86, which has a large size $\left(\alpha_{\mathrm{FS}}=42^{\prime}\right)$ and Fe-rich SN ejecta (Vink et al. 1997; Rho et al. 2002). This SNR has been associated with the supernova of AD 185 (Vink et al. 2006), but this association remains controversial, and its Type Ia origin is uncertain. If we ignore these concerns and take the value of $D=2.8 \mathrm{kpc}$ given by Rosado et al. (1996), we find $R_{\mathrm{FS}}=1.1 \times 10^{20} \mathrm{~cm}$ at an age of $1821 \mathrm{yr}$, about $30 \%$ larger than model PDDe $+\mathrm{L} 2$, but certainly within the correct order of magnitude. Recent values for the FS velocity $\left(\sim 2700 \mathrm{~km} \mathrm{~s}^{-1}\right.$; Vink et al. 2006) and the ionization timescale of the shocked ejecta $\left(\sim 10^{9} \mathrm{~cm}^{-3} \mathrm{~s}\right.$; Rho et al. 2002) are much closer to our cavity models PDDe $+\mathrm{HP} 3$ and PDDe $+\mathrm{L} 2$ than those of the SNRs in our sample. In fact, Vink et al. (1997) already suggested that RCW 86 might be evolving inside a lowdensity cavity based on early $A S C A$ observations. If the explosion type and the association with SN 185 are confirmed for RCW 86, it would be very interesting to explore the possibility of an accretion wind outflow as the origin of the CSM structure. Another Galactic SNR with Fe-rich ejecta that might be expanding into a CSM cavity is W49B (Hwang et al. 2000; Keohane et al. 2007), but in this case there is no reliable age estimate that can be used to constrain the dynamics.

\subsection{Implications of Accretion Wind Outflows for the Observations of Type Ia SNe}

Although this paper focuses on the impact that the outflows from Type Ia progenitors have on the dynamics and X-ray emission of SNRs, here we will briefly discuss the implications for observations of Type Ia SNe. The lack of prompt emission at $\mathrm{X}$-ray and radio wavelengths (Panagia et al. 2006; Immler et al. 2006 and other references listed in $\S 1$ ), as well as the absence of low-velocity (narrow) lines from $\mathrm{H}$ in the early or late spectra of normal events (Mattila et al. 2005), suggests that the amount of circumstellar material in the immediate vicinity of Type Ia progenitors must be low (Eck et al. 2002). The upper limits on the radio and X-ray fluxes have been used to derive estimates for the mass-loss rate of the progenitor system outflows $\dot{M}_{\text {of }}$, but these observations can only constrain the quotient $\dot{M}_{\mathrm{of}} / v_{\mathrm{of}}$. If the outflows are fast, this technique does not provide much information about $\dot{M}_{\mathrm{of}}$ : the radio fluxes of Panagia et al. (2006) translate into $\dot{M}_{\text {of }} \lesssim 3 \times 10^{-6} M_{\odot} \mathrm{yr}^{-1}$ for $v_{\text {of }}=1000 \mathrm{~km} \mathrm{~s}^{-1}$, which is compatible with all but the most extreme of accretion winds. For slower outflows, the upper limits on $\dot{M}_{\text {of }}$ go down linearly with $v_{\text {of }}$. An interesting possibility, suggested by WoodVasey \& Sokoloski (2006), is that episodic outflows just before the explosion might clear out a more or less extended region around the progenitor system, thus explaining the lack of prompt emission and H-rich material. However, we have shown that this region cannot be so large that the dynamic evolution of the SNR is severely affected, at least in the seven objects that we have studied. At the same time, evidence for large CSM structures around some Type Ia progenitors has appeared in the form of the light echoes detected in three objects: SN 1991T, SN 1995E, and SN 1998bu. These echoes have been interpreted by Quinn et al. (2006) as reflected light from detached shells or sheets of dust at distances ranging between 50 and $200 \mathrm{pc}$ from the SN. If this interpretation is correct (see Patat 2005 for a complete discussion of the ambiguities involved), the detached structures in these three objects might be the radiatively cooled shells around accretion-wind-blown bubbles.

\section{CONCLUSIONS}

In this paper, we have explored the relationship between the SD progenitor systems of Type Ia SNe and their surroundings, focusing on the effects that the outflows from these systems would have on the structure of the CSM and on the dynamics and X-ray emission of the SNRs that evolve after the explosion. We have seen that the optically thick accretion winds from the WD surface invoked by current Type Ia progenitor models will excavate large cavities in the ISM, provided that the outflows stay in the fast regime $\left(v_{\text {of }} \gtrsim 200 \mathrm{~km} \mathrm{~s}^{-1}\right)$. We have shown that the fundamental properties of the seven young Type Ia SNRs in our sample (SN 1885, Kepler, Tycho, SN 1006, 0509-67.5, 0519-69.0, and N103B) are incompatible with SNR models that expand into such large cavities. In fact, we found that all these objects can be explained by a uniform ISM interaction at the level of detail allowed by our simulations. At the same time, we cannot discard the existence of a population of young Type Ia SNRs expanding into low-density cavities excavated by accretion wind outflows. The properties of these objects (large size and low brightness) would make them hard to detect and, if detected, very difficult to identify as the remnants of Type Ia explosions. The Galactic SNR RCW 86 might be such an object, but more detailed work is needed to confirm this intriguing possibility.

The presence of outflows from the progenitors of the seven SNRs that we have examined cannot be completely discarded, 
but the properties of these outflows (mass-loss rates, timescales, and velocities) must be very different from those of the accretion winds described by HKN96. It remains to be seen whether outflows with substantially lower values of $\dot{M}_{\text {of }}$ or $v_{\text {of }}$ would be capable of limiting the effective accretion rate of the WD and prevent a common-envelope phase in single-degenerate Type Ia progenitors. Another possibility is that accretion wind outflows are not always present in these systems - for instance, if the rotation of the WD increases the accretion efficiency to the point that only a small fraction of systems present significant outflows, as proposed by Yoon \& Langer (2005).

We conclude with a reminder that the viability of SD systems as Type Ia progenitors has not been proved yet. Several studies have pointed out the difficulties in driving a $\mathrm{H}$-accreting WD close enough to the Chandrasekhar mass (Cassisi et al. 1998; Piersanti et al. 1999, 2000). If the star found by Ruiz-Lapuente et al. (2004) in the Tycho SNR is indeed the runaway companion of the WD that exploded in 1572, this means that at least one of the objects in our sample must have had a SD progenitor. The obvious alternative (DD systems) is plagued by its own problems, and it is still unclear whether WD mergers can lead to healthy explosions (Segretain et al. 1997; Guerrero et al. 2004). The fact remains that we lack a clear picture of the binary evolution leading to Type Ia
SNe. Studies of the CSM structure in young Type Ia SNRs should provide valuable insights into this problem.

We are very grateful to John Blondin for his assistance in setting up the VH-1 code, and for many discussions and valuable insights regarding the hydrodynamic simulations presented here. Kazik Borkowski provided us with several suggestions, including the importance of stellar motions and the relevance of RCW 86. We also acknowledge fruitful discussions on diverse topics with Martin Laming, Steve Reynolds, Jeno Sokoloski, Craig Wheeler, Philipp Podsiadlowski, and Garrelt Mellema. Support for this work was provided by the National Aeronautics and Space Administration through Chandra Postdoctoral Fellowship award PF6-70046 issued by the Chandra X-Ray Observatory Center, which is operated by the Smithsonian Astrophysical Observatory for and on behalf of the National Aeronautics and Space Administration under contract NAS8-03060. J. P. H. acknowledges support from Chandra grant G06-7016B. E. B. has received support from the DURSI of the Generalitat de Catalunya and the Spanish DGICYT grants AYA 2004-06290-C0202 and AYA 2005-08013-C03-01.

\section{APPENDIX}

\section{IONIZATION TIMESCALES FOR THE SHOCKED EJECTA IN TYPE Ia SUPERNOVA REMNANTS}

Together with the electron temperature $T_{e}$, the ionization timescale or fluence $\left(n_{e} t\right)$ is a fundamental quantity used to characterize the thermal emission in nonequilibrium ionization plasmas. These two parameters are the most common end product of fitting the thermal component of the X-ray emission in SNRs. However, the values of $T_{e}$ and $n_{e} t$ obtained in a particular spectral fit are model dependent, and can be affected by uncertainties in the atomic data and other factors (for discussions, see Borkowski et al. 2001; Rakowski et al. 2006). The most popular tools for spectral analysis of the thermal emission in SNRs are the plane-parallel shock models with adjustable abundances, but even these sophisticated spectral models contain fundamental simplifications whose impact on the fitted parameters is unclear, such as the assumption of a homogeneous chemical composition or the plane shock geometry. In general, it can be said that the systematic uncertainties associated with plane-parallel shock fits are much larger (and much harder to estimate) than the statistical ones.

In order to minimize these sources of error and determine values of $n_{e} t$ in different SNRs that can be compared to each other (and to the models) with some confidence, we have downloaded archival Chandra observations for the objects in our sample, and we have fitted them with the same procedure. We used the plane-parallel shock models described in Hughes et al. (2000) for the thermal component and a power law to describe the nonthermal continuum, both of which were absorbed by an intervening column of Galactic interstellar matter assumed to be of solar composition. During the fits, the Galactic column density was fixed to a value determined from the entire Chandra spectrum. To minimize the errors related to atomic data, we concentrated on the K-shell lines from the most abundant elements (spectra above $1.5 \mathrm{keV}$ ). This has the advantage of reducing the impact of uncertainties in the absorption as well. In fits to the $1.5-5 \mathrm{keV}$ band ("Si component"), we included emission from $\mathrm{Si}, \mathrm{S}, \mathrm{Ar}$, and $\mathrm{Ca}$, all with the same $T_{e}$ and $n_{e} t$. The relative abundances of these species were allowed to vary during the fit, as were the index and normalization of the power law. Independent fits were done to the 5$10 \mathrm{keV}$ band around the Fe K blend ("Fe component"). Only Fe and the power-law continuum (plus fixed absorption) were included in the Fe fits. The best-fit models for each SNR in both bands can be seen in Figure 10.

The relationship between the two fundamental quantities $n_{e} t$ and $T_{e}$ in these fits is of particular interest. Plane-parallel shock models implicitly rely on the thermal continuum to constrain $T_{e}$ and the line emission to constrain $n_{e} t$. Unfortunately, the X-ray continuum is often dominated by nonthermal emission (the power law in our fits), and the value of $T_{e}$ is largely unconstrained. We have taken this situation into account by producing fits at fixed values of $T_{e}: 1$ and $10 \mathrm{keV}$ for the Si component, 5 and $10 \mathrm{keV}$ for the Fe component. These values represent reasonable maxima and minima for shock-heated plasma in young SNRs, and the best-fit value for $T_{e}$ (models plotted in Fig. 10) is bounded by them in all cases. The range of ionization timescales listed in Table 4 corresponds to these extrema. We have also subtracted 0.3 dex from the fitted values to account for the fact that the plane-parallel shock models contemplate a distribution of ionization timescales between 0 and $n_{e} t$, so the average $\left(\left\langle n_{e} t\right\rangle\right)$ is one-half of the fitted value.

A rough idea of the level of agreement between our hydrodynamic models and the spectral fits to the observations can be obtained by comparing the fitted values of $\log \left(\left\langle n_{e} t\right\rangle\right)$ in the Tycho SNR from Table 4 (10.22-10.99 for Si, 9.72-9.78 for Fe) with the values of $\log (\langle\tau\rangle)$ in the best model for the ejecta emission found by Badenes et al. (2006) (10.12 for Si, 9.69 for Fe). These small deviations support the validity of the comparisons made in $\S 5.3$.

We conclude with an unrelated (but important) remark on the fitted ionization timescales listed in Table 4. In all the Type Ia SNRs we have examined, the $\left\langle n_{e} t\right\rangle$ of $\mathrm{Si}$ is significantly higher than that of Fe, sometimes by more than an order of magnitude. This constitutes strong evidence that the ejecta of Type Ia SNe must be stratified to some degree (with most of the Fe interior to most of the Si), favoring delayed detonation models over deflagration models with well-mixed ejecta (see discussion in $\S 3$ of Badenes et al. 2005). 

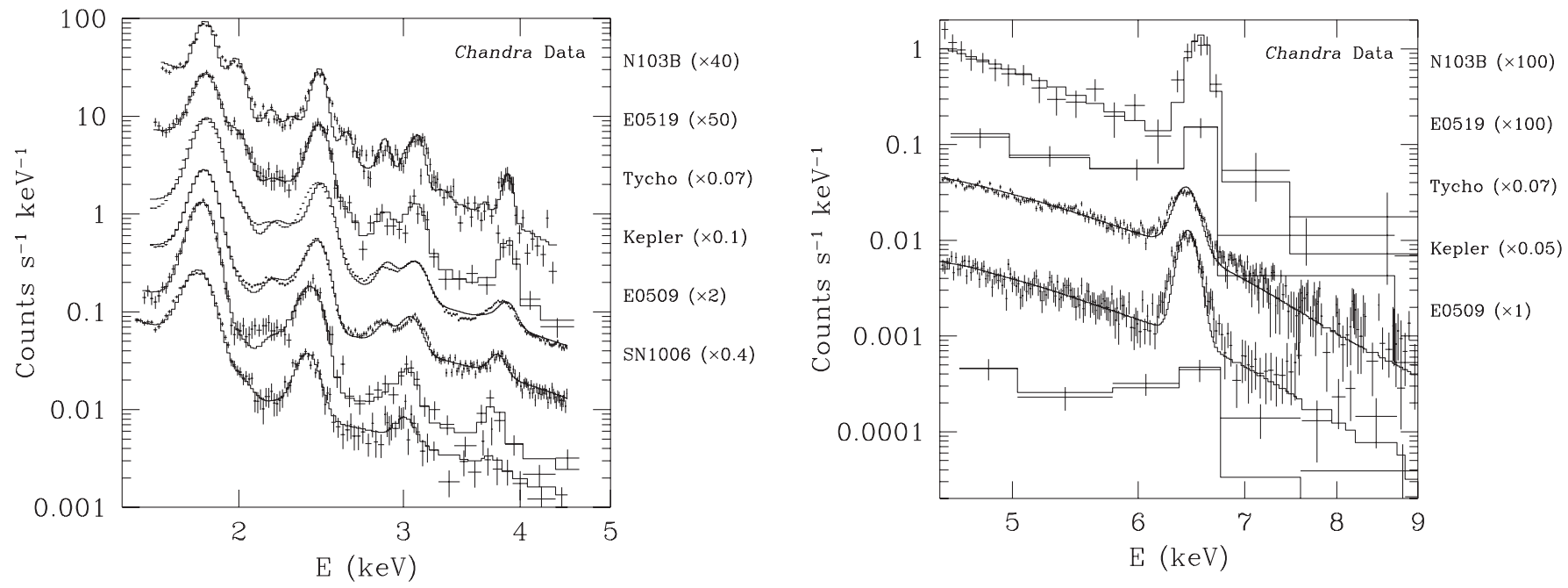

FIG. 10.- Plane-parallel shock fits to the Chandra spectra of the SNRs in the sample. The K-shell emission blends from Si, S, Ar, and Ca are shown in the left panel, and the Fe $\mathrm{K}$ blend is shown in the right panel. The spectra are roughly ordered from highest (top) to lowest (bottom) ionization timescale.

Alves, D. R. 2004, NewA Rev., 48, 659

Badenes, C. 2004, Ph.D. thesis, Univ. Politècnica Catalunya

Badenes, C., Borkowski, K. J., \& Bravo, E. 2005, ApJ, 624, 198

Badenes, C., Borkowski, K. J., Hughes, J. P., Hwang, U., \& Bravo, E. 2006, ApJ, 645, 1373

Badenes, C., \& Bravo, E. 2001, ApJ, 556, L41 (BB01)

Badenes, C., Bravo, E., Borkowski, K. J., \& Domínguez, I. 2003, ApJ, 593, 358

Balick, B., \& Frank, A. 2002, ARA\&A, 40, 439

Bandiera, R. 1987, ApJ, 319, 885

Borkowski, K. J., Blondin, J. M., \& Sarazin, C. L. 1992, ApJ, 400, 222

Borkowski, K. J., Hendrick, S. P., \& Reynolds, S. P. 2006, ApJ, 652, 1259

Borkowski, K. J., Lyerly, W. J., \& Reynolds, S. P. 2001, ApJ, 548, 820

Branch, D., Livio, M., Yungelson, L., Boffi, F., \& Baron, E. 1995, PASP, 107, 1019

Cassam-Chenai, G., Decourchelle, A., Ballet, J., Hwang, U., Hughes, J., \& Petre, R. 2004, A\&A, 414, 545

Cassisi, S., Iben, I., \& Tornambé, A. 1998, ApJ, 496, 376

Castor, J., McCray, R., \& Weaver, R. 1975, ApJ, 200, L107

Chevalier, R. A., \& Plait, P. C. 1988, ApJ, 331, L109

Chevalier, R. A., \& Raymond, J. C. 1978, ApJ, 225, L27

Colella, P., \& Woodward, P. 1984, J. Comput. Phys., 54, 174

Dalgarno, A., \& McCray, R. A. 1972, ARA\&A, 10, 375

Decourchelle, A., Ellison, D., \& Ballet, J. 2000, ApJ, 543, L57

Dehnen, W., \& Binney, J. J. 1998, MNRAS, 298, 387

Dere, K. P., Landi, E., Mason, H. E., Monsignori Fossi, B. C., \& Young, P. R. 1997, A\&AS, 125, 149

Deutschmann, A. 1998, M.A. thesis, Univ. Potsdam

de Vaucouleurs, G., \& Corwin, H. G., Jr. 1985, ApJ, 295, 287

Dwarkadas, V. V. 2005, ApJ, 630, 892

Eck, C. R., Cowan, J. J., \& Branch, D. 2002, ApJ, 573, 306

Ellison, D., Decourchelle, A., \& Ballet, J. 2004, A\&A, 413, 189

Ferrière, K. M. 2001, Rev. Mod. Phys., 73, 1031

Fesen, R. A., Hoeflich, P. A., Hamilton, A. J. S., Hammell, M. C., Gerardy, C. L., Khokhlov, A. M., \& Wheeler, J. C. 2007, ApJ, 658, 396

Ghavamian, P., Raymond, J., Smith, R., \& Hartigan, P. 2001, ApJ, 547, 995

Guerrero, J., García-Berro, E., \& Isern, J. 2004, A\&A, 413, 257

Hachisu, I., \& Kato, M. 2001, ApJ, 558, 323

2003a, ApJ, 598, 527

. 2003b, ApJ, 590, 445

Hachisu, I., Kato, M., Kato, T., Matsumoto, K., \& Nomoto, K. 2000, ApJ, 534, L189

Hachisu, I., Kato, M., \& Nomoto, K. 1996, ApJ, 470, L97 (HKN96) 1999a, ApJ, 522, 487

Hachisu, I., Kato, M., Nomoto, K., \& Umeda, H. 1999b, ApJ, 519, 314

Han, Z., \& Podsiadlowski, P. 2004, MNRAS, 350, 1301

Heng, K., \& McCray, R. 2007, ApJ, 654, 923

Hillebrandt, W., \& Niemeyer, J. 2000, ARA\&A, 38, 191

Hoyle, F., \& Fowler, W. 1960, ApJ, 132, 565

Hughes, J. P., Ghavamian, P., Rakowski, C. E., \& Slane, P. O. 2003, ApJ, 582, L95
REFERENCES

Hughes, J. P., Rakowski, C. E., \& Decourchelle, A. 2000, ApJ, 543, L61

Hughes, J. P., et al. 1995, ApJ, 444, L81

Hutchings, J. B., Winter, K., Cowley, A. P., Schmidtke, P. C., \& Crampton, D. 2002, AJ, 124, 2833

Hwang, U., Petre, R., \& Hughes, J. P. 2000, ApJ, 532, 970

Iben, I. J., \& Livio, M. 1993, PASP, 105, 1373

Immler, S., et al. 2006, ApJ, 648, L119

Ivanova, N., \& Taam, R. E. 2004, ApJ, 601, 1058

Kahabka, P., \& van den Heuvel, E. P. J. 1997, ARA\&A, 35, 69

Kato, M., \& Hachisu, I. 1994, ApJ, 437, 802 2004, ApJ, 613, L129

Keohane, J. W., Reach, W. T., Rho, J., \& Jarrett, T. H. 2007, ApJ, 654, 938

Kobayashi, C., Tsujimoto, T., Nomoto, K., Hachisu, I., \& Kato, M. 1998, ApJ, 503, L155

Koo, B.-C., \& McKee, C. F. 1992a, ApJ, 388, 93 1992b, ApJ, 388, 103

Landi, E., Del Zanna, G., Young, P. R., Dere, K. P., Mason, H. E., \& Landini, M. 2006, ApJS, 162, 261

Langer, N., Deutschmann, A., Wellstein, S., \& Höflich, P. 2000, A\&A, 362, 1046

Lewis, K., Burrows, D., Hughes, J., Slane, P., Garmire, G., \& Nousek, J. 2003, ApJ, 582, 770

Li, X., \& van den Heuvel, E. 1997, A\&A, 322, L9

Lucy, L. B., \& Abbott, D. C. 1993, ApJ, 405, 738

Mattila, S., Lundqvist, P., Sollerman, J., Kozma, C., Baron, E., Fransson, C., Leibundgut, B., \& Nomoto, K. 2005, A\&A, 443, 649

Moffett, D. A., Goss, W. M., \& Reynolds, S. P. 1993, AJ, 106, 1566

Nomoto, K. 1982, ApJ, 253, 798

Nomoto, K., \& Kondo, Y. 1991, ApJ, 367, L19

Nomoto, K., Saio, H., Kato, M., \& Hachisu, I. 2007, ApJ, in press (astro-ph/ 0603351)

Owocki, S. P., Gayley, K. G., \& Shaviv, N. J. 2004, ApJ, 616, 525

Pakull, M. W., Moch, C., Bianchi, L., Thomas, H.-C., Guibert, J., Beaulieu, J. P., Grison, P., \& Schaeidt, S. 1993, A\&A, 278, L39

Panagia, N., Van Dyk, S. D., Weiler, K. W., Sramek, R. A., Stockdale, C. J., \& Murata, K. P. 2006, ApJ, 646, 369

Park, S., Slane, P. O., Hughes, J. P., Mori, K., Burrows, D. N., \& Garmire, G. P. 2006, AAS HEAD Meeting, 9, 1.06

Patat, F. 2005, MNRAS, 357, 1161

Piersanti, L., Cassisi, S., Iben, I., Jr., \& Tornambé, A. 1999, ApJ, 521, L59 2000, ApJ, 535, 932

Quinn, J. L., Garnavich, P. M., Li, W., Panagia, N., Riess, A., Schmidt, B. P., \& Della Valle, M. 2006, ApJ, 652, 512

Raga, A. C., Mellema, G., \& Lundqvist, P. 1997, ApJS, 109, 517

Rakowski, C. E., Badenes, C., Gaensler, B. M., Gelfand, J. D., Hughes, J. P., \& Slane, P. O. 2006, ApJ, 646, 982

Rest, A., et al. 2005, Nature, 438, 1132

Reynolds, S. P., Borkowski, K. J., Hwang, U., Hughes, J. P., Badenes, C., Laming, J. M., \& Blondin, J. M. 2007, ApJ, submitted

Reynoso, E. M., \& Goss, W. M. 1999, AJ, 118, 926

Rho, J., Dyer, K., Borkowski, K., \& Reynolds, S. 2002, ApJ, 581, 1116 
Rosado, M., Ambrocio-Cruz, P., Le Coarer, E., \& Marcelin, M. 1996, A\&A, 315,243

Rózyczka, M., Tenorio-Tagle, G., Franco, J., \& Bodenheimer, P. 1993, MNRAS, 261, 674

Ruiz-Lapuente, P., et al. 2004, Nature, 431, 1069

Schlegel, E., \& Petre, R. 1993, ApJ, 412, L29

Segretain, L., Chabrier, G., \& Mochkovitch, R. 1997, ApJ, 481, 355

Smith, N., \& Owocki, S. P. 2006, ApJ, 645, L45

Smith, R., Kirshner, R., Blair, W., \& Winkler, P. 1991, ApJ, 375, 652

Sollerman, J., Ghavamian, P., Lundqvist, P., \& Smith, R. C. 2003, A\&A, 407, 249

Starrfield, S., Timmes, F. X., Hix, W. R., Sion, E. M., Sparks, W. M., \& Dwyer, S. J. 2004, ApJ, 612, L53

Sutherland, R., \& Dopita, M. 1993, ApJS, 88, 253

Tenorio-Tagle, G., Bodenheimer, P., Franco, J., \& Rózyczka, M. 1990, MNRAS, 244, 563

Tenorio-Tagle, G., Rózyczka, M., Franco, J., \& Bodenheimer, P. 1991, MNRAS, 251, 318

Tout, C. A. 2005, in ASP Conf. Ser. 330, The Astrophysics of Cataclysmic Variables and Related Objects, ed. J.-M. Hameury \& J.-P. Lasota (San Francisco: ASP), 279
Uenishi, T., Nomoto, K., \& Hachisu, I. 2003, ApJ, 595, 1094 Vink, J., Bleeker, J., van der Heyden, K., Bykov, A., Bamba, A., \& Yamazaki, R. 2006, ApJ, 648, L33

Vink, J., Kaastra, J. S., \& Bleeker, J. A. M. 1997, A\&A, 328, 628

Warren, J., \& Hughes, J. 2004, ApJ, 608, 261

Warren, J., et al. 2005, ApJ, 634, 376

Weaver, R., McCray, R., Castor, J., Shapiro, P., \& Moore, R. 1977, ApJ, 218, 377

Winkler, P. F., Gupta, G., \& Long, K. S. 2003, ApJ, 585, 324

Wood, J. H., \& Lockley, J. J. 2000, MNRAS, 313, 789

Wood-Vasey, W. M., \& Sokoloski, J. L. 2006, ApJ, 645, L53

Yaron, O., Prialnik, D., Shara, M. M., \& Kovetz, A. 2005, ApJ, 623, 398

Yoon, S.-C., \& Langer, N. 2004, A\&A, 419, 645 2005, A\&A, 435, 967

Yoon, S.-C., Langer, N., \& Scheithauer, S. 2004a, A\&A, 425, 217

Yoon, S.-C., Langer, N., \& van der Sluys, M. 2004b, A\&A, 425, 207 